\title{
Dinâmica de homeomorfismos homotópicos à Dehn twists
}

\author{
Bráulio Augusto Garcia
}

TESE APRESENTADA

AO

Instituto de Matemática e Estatística

DA

Universidade de São Paulo

PARA

OBTENÇÃO DO TÍTULO

$\mathrm{DE}$

Doutor em Cî̂ncias

Programa: Matemática Aplicada

Orientador: Prof. Dr. Salvador Addas Zanata

Durante o desenvolvimento deste trabalho o autor recebeu auxílio financeiro da

FAPESP

São Paulo, fevereiro de 2012 


\title{
Dinâmica de homeomorfismos homotópicos à Dehn twists
}

\begin{abstract}
Esta tese contém as correções e alterações sugeridas pela Comissão Julgadora durante a defesa realizada por Bráulio Augusto Garcia em 02/02/2012. O original encontra-se disponível no Instituto de Matemática e Estatística da Universidade de São Paulo.
\end{abstract}

Banca Examinadora:

- Prof. Dr. Salvador Addas Zanata (orientador) - IME-USP.

- Prof. Dr. Fabio Armando Tal - IME-USP.

- Prof. Dr. Pedro Antonio Santoro Salomão - IME-USP.

- Prof. Dr. Andrés Koropecki- UFF.

- Prof. Dr. Mário Jorge Dias Carneiro - UFMG. 


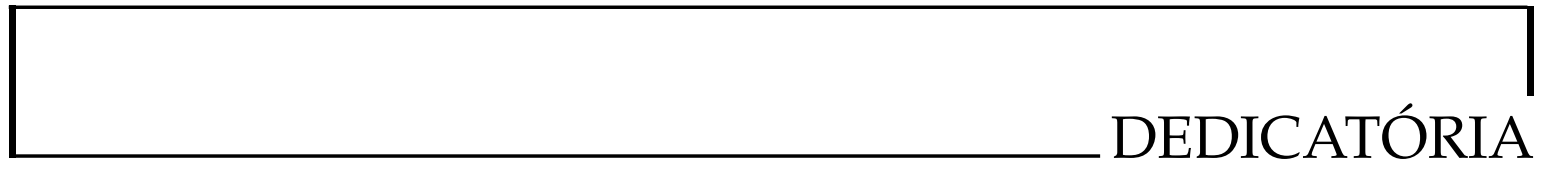

Dedico essa tese aos meus pais. 
Agradeço...

A minha família por compreender e perdoar minha ausência.

Ao meu amor Ira por estar sempre ao meu lado, por contribuir muito em minha vida (desde o início da graduação) e por me deixar cada dia mais feliz. Te amo.

Ao meu amigo-irmão Felipe Chaves, pelas conversas sobre o universo, Raul Seixas, etc., nas montanhas de Minas, onde iniciamos nossos estudos sérios em física e depois em matemática.

Ao meu orientador Dr. Salvador, a quem posso chamar de amigo e a quem tenho uma dívida impagável, por me introduzir na dinâmica topológica, por ser um ótimo professor, pesquisador, etc., e, principalmente, por ser uma pessoa de grande humildade e honestidade, tanto academicamente quanto pessoalmente. Gostaria de dizer que me sinto uma pessoa privilegiada de ter te conhecido e de ter trabalhado em minha tese sob sua orientação. Obrigado pelas caminhadas no campus da USP, onde realizávamos a pesquisa sobre Dehn twists.

Ao professor Dr. Fábio Tal, por participar dessas caminhadas pela USP, por sempre estar disponível para conversar sobre matemática e por ter dado grandes ideias na elaboração da tese.

Aos professores Dr. Andre de Carvalho e Dr. Philip Boyland pelo excelente curso sobre teoria de Nielsen-Thurston.

Ao meu amigo e também companheiro de sala Valentim, pelas discussões sobre matemática, sistemas dinâmicos, física, literatura (brasileira, espanhola, ...) e a vida. Também a Sebastiam por estar muitas vezes nessas discussões.

À minha amiga Tatiane, por ser companheira de café (mesmo ela sempre tomando 
chá) e aos meus outros amigos do IME e CBPF, Lucas, Antônio, Anderson, Pricila, Gustavo, Humberto, Grasi, Bittencourt...

Aos funcionários e professores do IME, pelo ambiente acolhedor proporcionado. Aos professores que me deram aula (da UNIFEI e do IME), pela dedicação e profissionalismo.

À Universidade de São Paulo, pela oportunidade de estudo e por toda a infraestrutura oferecida.

À UNIFEI, por ter sido meu berço acadêmico. Bem como aos meus primeiros mestres de graduação, Baêta Segundo, Luis Fernando e Vitório De Lorenci.

Aos professores (Clodoaldo, Saulo, ...) e colegas (Jaime, Borba, ...) do futebol.

Sou grato por ter vivido com esses profissionais que levam o ensino público a sério, o que só reforça minhas convicções.

Ao pessoal da república, que foram minha família aqui em São Paulo, Danilo, Maguinho e Fabrício, companheiros de todas as horas!

À FAPESP, pelo apoio financeiro, que é fundamental numa cidade como São Paulo.

A todos aqueles que contribuíram, mesmo que indiretamente, na realização desse trabalho. Muito obrigado! 
Amanhece, amanhece, amanhece, amanhece, amanhece o dia Um leve toque de poesia Com a certeza que a luz que se derrama nos traga um pouco, um pouco, um pouco de alegria! A frieza do relógio não compete com a quentura do meu coração Coração que bate 4 por 4 sem lógica e sem e sem nenhuma razão Bom dia sol !!! Bom dia, dia! Coração Noturno: Raul Seixas. 
Dinâmica de homeomorfismos homotópicos à Dehn twists.

No presente trabalho apresentamos um estudo sobre a dinâmica de homeomorfismos do toro homotópicos à Dehn twists. No caso conservativo, provamos que se $f$ preserva área e tem um levantamento $\hat{f}$ para o cilindro com fluxo zero, então, precisamente, ou $f$ é um homeomorfismo do anel, ou possui pontos no cilindro com velocidades verticais positiva e negativa, por iteradas de $\hat{f}$. Isso resolve a conjectura de Boyland para essa classe de homotopia. Já no caso geral, mostramos um resultado análogo. Além disso, fornecemos uma condição extremamente simples que, quando satisfeita, implica que o conjunto de rotação vertical contém um intervalo e, portanto, que $f$ tem entropia topológica positiva.

Palavras-chave: conjunto de rotação vertical, Dehn twists, omega limites, decomposição por tijolos. 
Dynamics of homeomorphisms homotopic to Dehn twists.

The present thesis is concerned with the dynamics of homeomorphisms of the torus homotopic to Dehn twists. We prove that if $f$ is area preserving and it has a lift $\hat{f}$ to the cylinder with zero flux, then either $f$ is an annulus homeomorphism, or there are points in the cylinder with positive vertical velocity and others with negative vertical velocity, for iterates of $\hat{f}$. This solves a version of Boyland's conjecture to this setting. We extend some theorems we already obtained for Dehn twists with the area preservation hypothesis to a more general class. Finally, we also give a simple explicit condition which, when satisfied, implies that the vertical rotation set contains an interval and thus also implies positive topological entropy.

Keywords: vertical rotation set, Dehn twists, omega limits, brick decompositions. 
1 Introdução 1

1.1 Considerações Preliminares . . . . . . . . . . . . . . . . . . 2

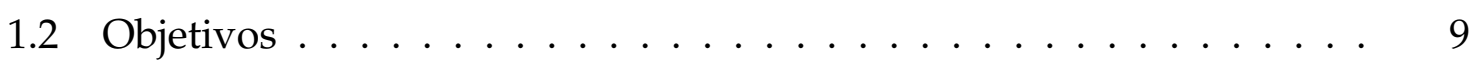

1.3 Contribuições . . . . . . . . . . . . . . . . . . . 10

$\begin{array}{lll}2 & \text { Fundamentos } & 12\end{array}$

2.1 O Conjunto de Rotação . . . . . . . . . . . . . . . . . . . . . . 12

2.2 O Conjunto de Rotação Vertical . . . . . . . . . . . . . . . . . . . . . . 14

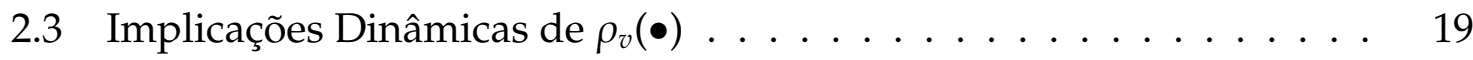

3 Ferramentas Básicas $\quad 22$

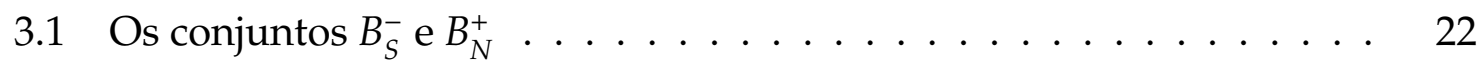

3.2 Os conjuntos $\omega$-limite de $B_{S}^{-}$e $B_{N}^{+} \ldots \ldots \ldots \ldots \ldots$. . . . . . . . . . . . . . . . . . . .

3.3 Homeomorfismos de Brouwer . . . . . . . . . . . . . . . . 39

3.4 Decomposição do plano por tijolos . . . . . . . . . . . . . . . 40

$4 \quad$ Provas dos principais resultados $\quad 42$

4.1 Demonstração do teorema 1.3.1 . . . . . . . . . . . . . . . . 42

4.2 Demonstração do teorema $1.3 .3 \ldots \ldots$. . . . . . . . . . . 55 
1.1 Ação de um Dehn twist $\ldots \ldots \ldots \ldots \ldots$. . . . . . . . . . 7

1.2 Dinâmica de um Dehn twist em $\mathbb{R}^{2} / \mathbb{Z}^{2} \ldots \ldots \ldots \ldots \ldots$

1.3 Dinâmica reduzida de $\hat{f}$ ao anel $\ldots \ldots \ldots \ldots . \ldots \ldots$

2.1 Levantamento $\hat{f}$ com fluxo zero . . . . . . . . . . . . . . . 18

2.2 Aplicações de recobrimento. . . . . . . . . . . . . . . . . . . 21

3.1 Dinâmica na compactificação . . . . . . . . . . . . . . 23

3.2 Arcos contínuos simples $\Gamma_{N} \mathrm{e} \bar{\Gamma}_{N} \ldots \ldots \ldots \ldots \ldots \ldots \ldots$

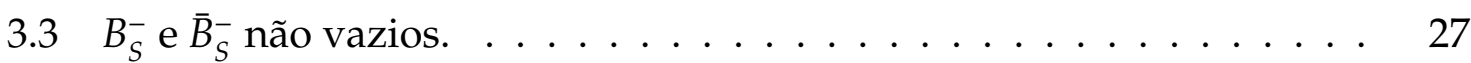

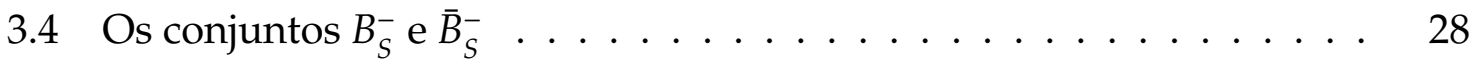

3.5 Órbitas uniformemente limitadas no cilindro . . . . . . . . . . . . . . 29

3.6 Órbitas ilimitadas em apenas uma direção do cilindro . . . . . . . . 30

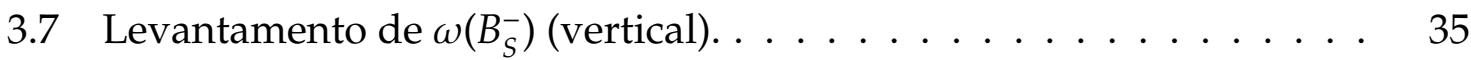

3.8 Levantamento de $\omega\left(B_{S}^{-}\right)$(horizontal). . . . . . . . . . . . . . . 36

3.9 Levantamento dos arcos contínuos simples. . . . . . . . . . . . . 37

$4.1 \quad$ Transladado vertical de $\pi^{-1}\left(\omega\left(B_{S}^{-}\right)\right) . \ldots \ldots \ldots \ldots \ldots \ldots$. . . . . 47

4.2 Decomposição do Plano por Tijolos Livres para $\tilde{g}$.

4.3 Violação do fluxo zero. . . . . . . . . . . . . . . . . . . . 56 


\section{CAPÍTULO 1}

INTRODUÇÃO

Um par ordenado da forma $(X, f)$ é dito ser um sistema dinâmico se $f$ é uma aplicação de $X$ em $X$, isto é, $f: X \longrightarrow X$. O conjunto $X$ é denominado espaço e $f$ dinâmica.

A órbita (futura) de um ponto de $X$ é dada por iterações da dinâmica, ou seja, se $x \in X$, então $\operatorname{orb}(x)=\left\{x, f(x), f(f(x)), \ldots, f^{n}(x), \ldots\right\}$, em palavras, uma espécie de movimento que evolui com o tempo (no caso, os números naturais) sob aplicação de uma lei repetidamente.

Nesse contexto, a pergunta chave é a existência de pontos periódicos para a dinâmica $f$, isto é, existência de $x_{f}$ tal que $\operatorname{orb}\left(x_{f}\right)=\left\{x_{f}, f\left(x_{f}\right), \ldots, f^{n-1}\left(x_{f}\right)\right\}$, para algum $n \in \mathbb{N}^{*}$. Um sistema dinâmico é uma abstração matemática que serve para modelar e fornecer previsões sobre inúmeros fenômenos naturais, sociais, econômicos e outros.

Quando X é uma superfície topológica e a aplicação $f$ um homeomorfismo sobre $X$, temos um sistema dinâmico topológico (inversível). Nesse caso, a questão mais geral que órbitas periódicas é a existência de conjuntos minimais, onde $K \subset X$ é minimal se, para todo $x \in K, \omega(x)=\cap_{i=1}^{\infty} \overline{\cup_{j=i}^{\infty} f^{j}(x)}=K$, ou seja, o omega limite de qualquer ponto de $K$ é todo $K$ (veja [24]). O estudo da dinâmica de homeomorfismos em superfícies por métodos topológicos provavelmente se iniciou com H. Poincaré, com seu "último teorema geométrico", hoje em dia mais conhecido como teorema de Poincaré-Birkhoff.

Um conceito que tem-se mostrado muito útil em sistemas dinâmicos é a noção de número de rotação, que foi introduzido por Poincaré no caso de um homeomorfismo do círculo que preserva orientação, bem como as definições e generalizações a partir 
desse. Para homeomorfismos do círculo, ele mostrou que todas as órbitas possuem o mesmo número de rotação e, juntamente com os trabalhos de Denjoy, esse número (o número de rotação do homeomorfismo) essencialmente classifica a dinâmica, sendo assim, um poderoso invariante topológico. De fato, quando este número é racional, sempre existem órbitas periódicas, todas com o mesmo período, e todas as órbitas são homoclínicas ou heteroclínicas às órbitas periódicas. Já no caso em que o número de rotação é irracional, não existem órbitas periódicas e todas as órbitas se "ordenam"como as órbitas de uma rotação irracional de mesmo número. Além disso, ou todas são densas, ou existem intervalos errantes e um conjunto minimal, onde todas as outras órbitas nascem e morrem, veja [27] e [26].

Esse conceito foi estendido para endomorfismos do círculo de grau um, por Newhouse, Palis e Takens, e para homeomorfismos de superfície como o anel e o toro, por M. Misiurewicz, K. Ziemian, J. Franks, e outros com a definição de intervalo e conjunto de rotação. Em termos grosseiros essa técnica permite medir, quando existir, a velocidade média da órbita de um ponto em rotação ao redor de buracos no espaço sob iteração da dinâmica. E, se o " conjunto de rotação tiver interior " então, temos que a dinâmica possui entropia topológica positiva, veja [24] e [29].

\subsection{Considerações Preliminares}

O objetivo da presente seção é motivar o estudo dos homeomorfismos homotópicos à Dehn twist no toro. Para isso, iniciemos com a classificação de homeomorfismos do toro. Primeiramente, considere o toro (plano) como sendo $\mathbb{R}^{2} / \mathbb{Z}^{2}$ e o denotemos por $\mathbb{T}^{2}$. O conjunto dos homeomorfismos de $\mathbb{T}^{2}, \operatorname{Homeo}\left(\mathbb{T}^{2}\right)$, pode ser particionado em, essencialmente, três classes de equivalência através da relação de homotopia entre aplicações do toro, a saber:

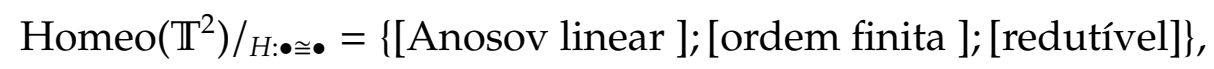

onde $H: \bullet \cong \bullet$ é a relação de homotopia, ou seja, $f, g \in \operatorname{Homeo}\left(\mathbb{T}^{2}\right)$ são tais que $H: f \cong g$ se, e somente se, existe uma aplicação (homotopia) $H: \mathbb{T}^{2} \times[0,1] \longrightarrow \mathbb{T}^{2}$ contínua e tal que $H_{0}(\bullet)=H(\bullet, 0)=f(\bullet)$ e $H_{1}(\bullet)=H(\bullet, 1)=g(\bullet)$. Se para todo $t \in] 0,1\left[, H_{t}: \mathbb{T}^{2} \longrightarrow \mathbb{T}^{2}\right.$ for um homeomorfismo, então $f$ é dita ser isotópica à $g$. 
Observação 1.1.1. Em superfícies fechadas o conceito de homotopia e isotopia entre aplicações coincidem, veja [21].

Essa classificação será discutida nos próximos parágrafos. Seja $f: \mathbb{T}^{2} \longrightarrow \mathbb{T}^{2}$ um homeomorfismo do toro, então, temos uma aplicação induzida no grupo fundamental do toro, da seguinte forma

$$
[\gamma] \in \pi_{1}\left(\mathbb{T}^{2}\right) \longmapsto[f \circ \gamma] \in \pi_{1}\left(\mathbb{T}^{2}\right)
$$

isto é, pela ação de $f$ nos laços de $\mathbb{T}^{2}$. Denotemos essa aplicação induzida por

$$
f_{*}: \pi_{1}\left(\mathbb{T}^{2}\right) \longrightarrow \pi_{1}\left(\mathbb{T}^{2}\right)
$$

Utilizando a estrutura aditiva de $\pi_{1}\left(\mathbb{T}^{2}\right)$, com sua base usual dado pelo isomorfismo natural $\pi_{1}\left(\mathbb{T}^{2}\right) \cong \mathbb{Z}^{2}$, podemos ver que $f_{*}$ é um isomorfismo e, portanto, pode ser representado como um elemento de $G L(2, \mathbb{Z})=\left\{A \in M_{2 \times 2}(\mathbb{Z}): \operatorname{det} A \neq 0\right\}$. Reciprocamente, se $A \in G L(2, \mathbb{Z})$, a transformação linear invertível $A: \mathbb{R}^{2} \longrightarrow \mathbb{R}^{2}$ se projeta a um homeomorfismo de $\mathbb{T}^{2}$, denotado por $\phi_{A}$. Pois, $A\left((\tilde{x}, \tilde{y})+\left(n_{1}, n_{2}\right)\right)=$ $A(\tilde{x}, \tilde{y})+\left(m_{1}, m_{2}\right)$ onde $(\tilde{x}, \tilde{y}) \in \mathbb{R}^{2}$ e $\left(n_{1}, n_{2}\right),\left(m_{1}, m_{2}\right) \in \mathbb{Z}^{2}$. Portanto, a aplicação $(\tilde{x}, \tilde{y}) \mapsto A(\tilde{x}, \tilde{y})$ passa ao quociente de $\mathbb{R}^{2}$ por $\mathbb{Z}^{2}$, produzindo um homeomorfismo $\phi_{A}$ do toro denominado automorfismo linear.

Não é difícil ver que todo $f \in \operatorname{Homeo}\left(\mathbb{T}^{2}\right)$ é homotópico a um automorfismo linear $\phi_{A}$, para algum $A \in G L(2, \mathbb{Z})$. Desse modo, a caracterização dos automorfismos lineares, $\phi_{A}$, fornece a classificação dos elementos de Homeo( $\left.\mathbb{T}^{2}\right)$. Mas esses são o conjunto $G L(2, \mathbb{Z}$ ), que é caracterizado pela função traço (ou pelos autovetores de $A$ ). Com efeito, seja $A \in G L(2, \mathbb{Z})$, então, $\operatorname{det} A=\mp 1$. Estamos interessados nos casos que preservam orientação, portanto, considere $A \in \operatorname{det}^{-1}(1) \subset G L(2, \mathbb{Z})$, ou seja, $A \in S L(2, \mathbb{Z})$ e tem autovalores dado por

$$
\lambda_{ \pm}=\frac{\operatorname{traço}(A) \pm \sqrt{\operatorname{traço}(A)^{2}-4}}{2} .
$$

Nessas condições, o traço de $A$ determina univocamente $\lambda_{ \pm}$no plano complexo e, consequentemente, fornece a partição de Homeo $\left(\mathbb{T}^{2}\right)$ nas ditas classes Anosov linear, ordem finita e redutível. Pois:

1. $|\operatorname{traço}(A)|>2, A$ tem autovalores $\lambda$ e $\lambda^{-1}$ com $|\lambda|>1$. Nesse caso, $A$ é dita hiperbólica e $\phi_{A}$ dita Anosov linear. 
2. $\operatorname{traço}(A) \in\{-1,0,1\}, A$ tem autovalores $\lambda$ e $\bar{\lambda} \operatorname{com} \lambda^{n}=1$, para algum $n \in\{3,4,6\}$ e portanto $A^{n}=I d$. Nesse caso $\phi_{A}$ é de ordem finita. Também, se traço $(A)= \pm 2$, $A$ é de ordem finita (com $n=1$ ou 2) se seu autovalor não tiver multiplicidade 2, ou seja, $A$ possui dois autovetores associado ao autovalor.

3. Caso contrário $A$ é dita redutível.

Pode-se mostrar que no último caso, a menos de mudanças de coordenadas, $A$ tem a forma

$$
A=\left[\begin{array}{ll} 
\pm 1 & m \\
0 & \pm 1
\end{array}\right]_{2 \times 2}
$$

onde $m \in \mathbb{Z}^{*}$. Nesse caso, a aplicação induzida $\phi_{A}$ recebe o nome de Dehn twist (ou twist de Dehn, ou Shear), veja [16] e [7]. Em suma, temos o seguinte teorema de caracterização:

Teorema 1.1.2. Seja $f: \mathbb{T}^{2} \longrightarrow \mathbb{T}^{2}$ um homeomorfismo. Então, $f$ é homotópico a, exatamente, um dos homeomorfismos abaixo:

1. Anosov linear;

2. Ordem finita;

3. Dehn twist.

A próxima proposição fornece algumas simples equivalências em termos da dinâmica no toro, grupo fundamental e no recobrimento.

Proposição 1.1.3. Seja $f: \mathbb{T}^{2} \longrightarrow \mathbb{T}^{2}$ um homeomorfismo. Então, são equivalentes:

1. $f$ é isotópico a $\phi_{A}$.

2. $f_{*}$ age sobre $H_{1}\left(\mathbb{T}^{2}\right) \cong \mathbb{Z}^{2}$ na base canônica pela matriz $A$.

3. Qualquer levantamento de $f, \tilde{f}$ para $\mathbb{R}^{2}$ (recobrimento universal) pode ser escrito como

$$
\tilde{f}(\tilde{x}, \tilde{y})=A(\tilde{x}, \tilde{y})+\gamma(\tilde{x}, \tilde{y}),
$$

$\operatorname{com} \gamma((\tilde{x}, \tilde{y})+(1,1))=\gamma(\tilde{x}, \tilde{y})$ 
4. Se $\tilde{f}$ é levantamento de $f$ para $\mathbb{R}^{2}$,

$$
\tilde{f}\left((\tilde{x}, \tilde{y})+\left(n_{1}, n_{2}\right)\right)=\tilde{f}(\tilde{x}, \tilde{y})+A\left(n_{1}, n_{2}\right),
$$

para todo $(\tilde{x}, \tilde{y}) \in \mathbb{R}^{2}$ e para todo $\left(n_{1}, n_{2}\right) \in \mathbb{Z}^{2}$.

No que segue, analisaremos as três classes de homeomorfismos do ponto de vista dinâmico.

Quando a matriz $A$ é hiperbólica, o seguinte teorema garante que homeomorfismos isotópicos à $\phi_{A}$ possuem "mais dinâmica" que $\phi_{A}$, em outras palavras, um Anosov linear tem a menor complexidade dinâmica em toda sua classe de isotopia. Esse teorema é frequentemente conhecido como teorema de (Franks [24]) estabilidade por isotopia sobre o toro.

Teorema 1.1.4. Se $f: \mathbb{T}^{2} \longrightarrow \mathbb{T}^{2}$ é um homeomorfismo isotópico a um Anosov linear, $\phi_{A}$, então existe uma aplicação contínua e sobrejetora $\alpha: \mathbb{T}^{2} \longrightarrow \mathbb{T}^{2}$ homotópica à identidade tal que $\alpha \circ f=\phi_{A} \circ \alpha$, ou como no diagrama comutativo:

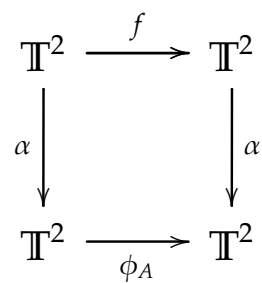

Sendo assim, $\phi_{A}$ é um fator de $f$ e, como é bem sabido, possui dinâmica bem interessante. Em outras palavras,

- possui entropia topológica positiva, isto é, $h_{t o p}\left(\phi_{A}\right)=\log |\lambda|$, onde $\lambda$ é o maior autovalor de $A$;

- possui órbitas densas, isto é, $\phi_{A}$ é transitiva;

- o conjunto dos pontos periódicos de $\phi_{A}$ é denso em $\mathbb{T}^{2}$; e

- possui folheações invariantes transversais densas sobre $\mathbb{T}^{2}$, sendo que uma delas expande e a outra contrai a dinâmica.

Já as aplicações induzidas por $A$ não hiperbólicas, ou seja, o raio espectral de $A$ é igual a 1 possuem dinâmicas triviais, pois são essencialmente rotações. Desse modo, possuem entropia topológica nula. Logo, só o fato de termos um homeomorfismo $f$ homotópico a um automorfismo linear não hiperbólico não nos diz nada dinamicamente, pois temos apenas que $h_{\text {top }}(f) \geq 0$. 
Assim, uma questão natural é sob que condições (dinâmicas, topológicas, etc) podemos garantir uma dinâmica não trivial?

\section{Homeomorfismos homotópicos à Dehn twists.}

A questão colocada anteriormente será explorada para a classe dos homeomorfismos homotópicos à Dehn twists, ou seja, queremos encontrar hipóteses topológicas e dinâmicas simples que produzam uma dinâmica complicada (rica) nessa classe.

O conjunto dos difeomorfismos twists (e tilts) do toro são exemplos particulares da classe Dehn twist e esses, como é bem sabido, possuem uma teoria muito importante na área de sistemas dinâmicos, bem como diversas aplicações em áreas afins. Essa condição de torção aparece com frequência em várias situações aparentemente não relacionadas. Por exemplo, o modelo de Frenkel-Kontorova, as geodésicas no toro, as pertubações periódicas de Hamiltonianos em dimensão dois, as aplicações do tipo bilhar em curvas convexas, os Hamiltonianos com dois graus de liberdade, em particular no problema restrito de três corpos, a dinâmica numa vizinhança de um ponto elíptico. Portanto, a teoria dos difeomorfismos do tipo twist pode ser considerada como um modelo teórico unificador para vários fenômenos. Veja [25], [23] e [24].

Sendo assim, estamos interessados e guiados por essa propriedade topológica de "entortar verticais" de um Dehn twist. No caso, de aplicações twists (ou tilts) uma restrição muito forte é imposta. E essa acarreta fortes restrições na dinâmica [14] e [2]. Desse modo, nosso interesse é o caso geral, ou seja, estudaremos somente os aspectos topológicos dessa propriedade, assim como suas imposições dinâmicas.

No próximo capítulo apresentaremos a estratégia que será usada para abordar esse problema. A figura seguinte é uma representação pictórica dessa propriedade e uma descrição de como definir um Dehn twist (ao longo de uma curva) no caso de superfícies de gênero maior. 


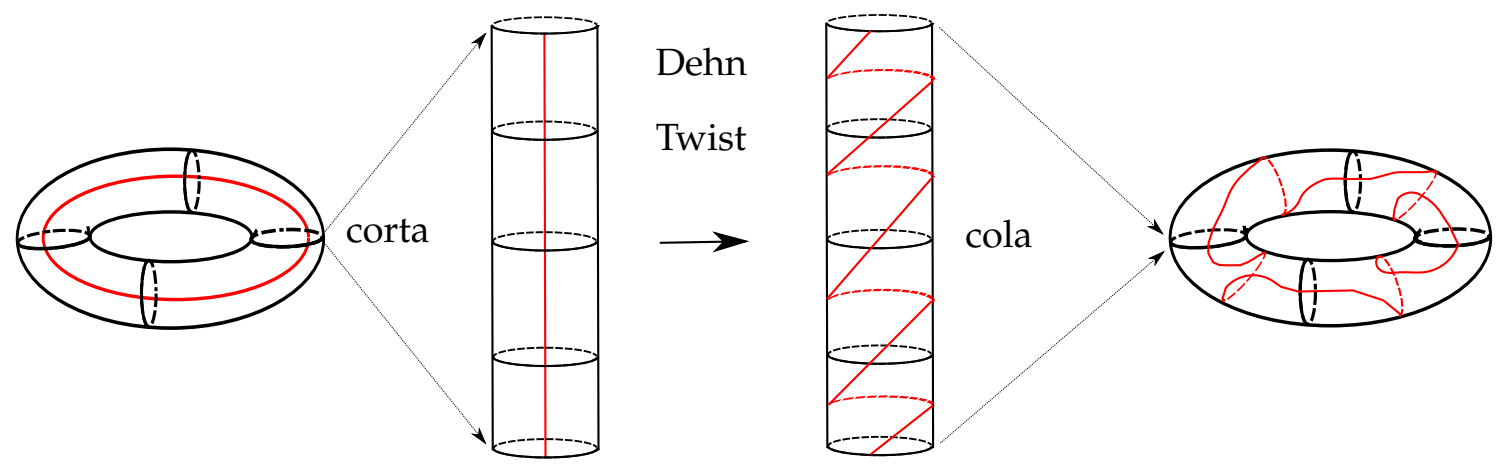

Figura 1.1: Ação de um Dehn twist

No caso do toro plano a dinâmica de um Dehn twist é exemplificado na figura abaixo.
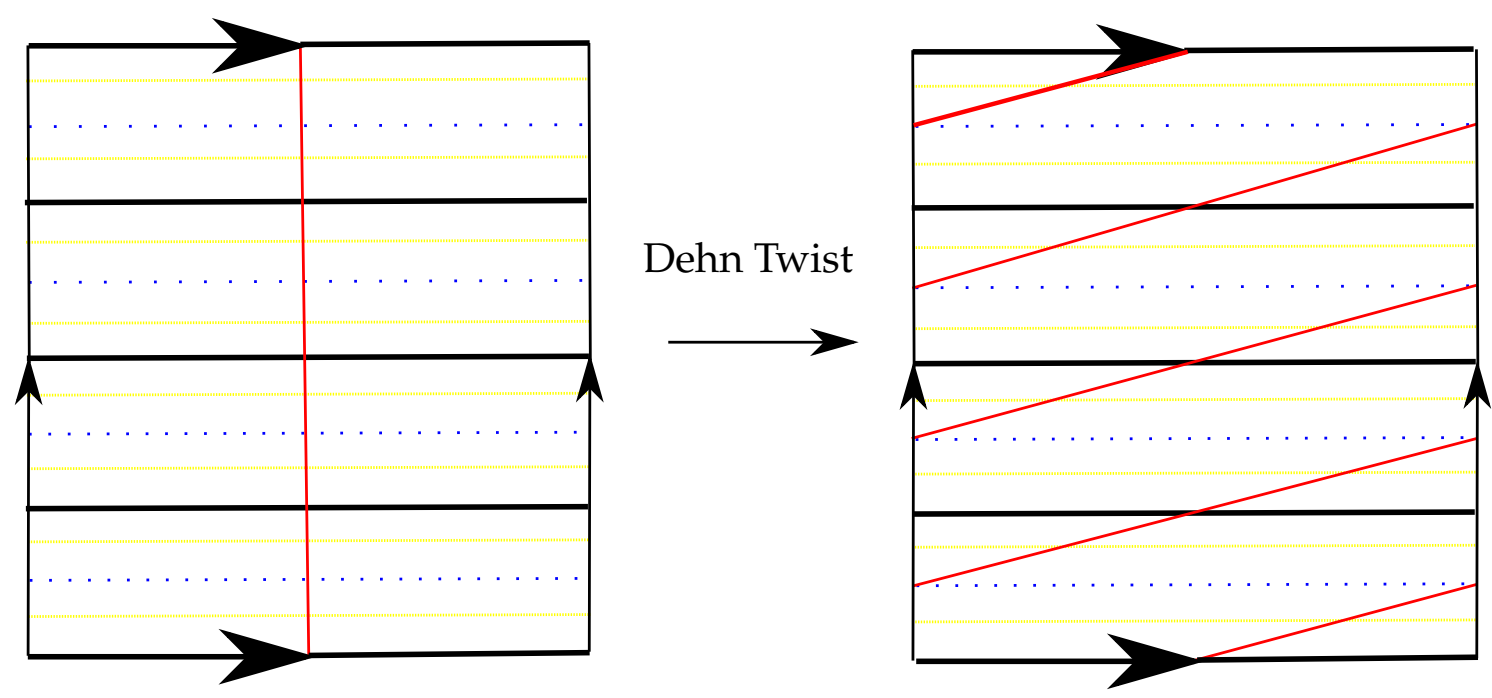

Figura 1.2: Dinâmica de um Dehn twist em $\mathbb{R}^{2} / \mathbb{Z}^{2}$ 
Este trabalho está organizado do seguinte modo:

Nas duas próximas seções, apresenta-se os objetivos e as contribuições desse estudo.

No capítulo 2, introduz-se a noção de conjunto de rotação para dinâmicas de homeomorfismos homotópicos à identidade e à Dehn twist. Apresenta-se algumas implicações dinâmicas fornecidas por essa teoria de rotação, e mostra-se que essas classes (identidade versus Dehn twist) possuem comportamentos diferentes.

No capítulo 3 (e em diante), estuda-se os homeomorfismos Dehn twists através de uma técnica de compactificação da dinâmica, adicionamos dois pontos fixos no infinito do recobrimento e analisamos o comportamento assintótico (o omega limite) de certos conjuntos a esses pontos. Demonstra-se que o omega limite de um conjunto específico fornece informações sobre o conjunto de rotação (vertical) e, consequentemente, restrições na dinâmica. Enuncia-se alguns importantes resultados da teoria de Brouwer para homeomorfismos do plano.

Finalmente, dedica-se o último capítulo as provas dos principais resultados desse trabalho. 


\subsection{Objetivos}

O objetivo desse trabalho é estudar no contexto das aplicações homotópicas à Dehn twists algumas das muitas importantes conjecturas feitas para aplicações homotópicas à identidade. Como por exemplo, a conjectura de Boyland :

Suponha que $f$ tenha fluxo zero (2.2.5) e que exista uma medida Boreleana de probabilidade $f$-invariante com número de rotação vertical positivo, então é verdade que existe um ponto com número de rotação vertical negativo?

Outras questões são: $\mathrm{O}$ conjunto dos $C^{r}$-difeomorfismos Dehn twists minimais possui interior vazio para $r \geq 2$ ?

Se $f$ é um homeomorfismo Dehn twist que preserva área e tem número de rotação vertical da medida de Lebesgue zero, então é verdade que ou $f$ é um homeomorfismo do anel, ou seu intervalo de rotação vertical possui interior não vazio?

Ou mais geral, se $f$ é um homeomorfismo Dehn twist cujo conjunto de rotação vertical contém o zero, então é verdade que ou $f$ possui um anel invariante, ou seu intervalo de rotação vertical não se reduz só ao zero?

Existe uma condição simples (dinâmica ou topológica) que, quando satisfeita por um homeomorfismo Dehn twist, implique que esse tenha entropia topológica positiva? 


\subsection{Contribuições}

Sejam $f: \mathbb{T}^{2} \longrightarrow \mathbb{T}^{2}$ um homeomorfismo homotópico a um Dehn twist e $\hat{f}: S^{1} \times \mathbb{R} \longrightarrow$ $S^{1} \times \mathbb{R}$ um levantamento de $f$ para o cilindro vertical. Nessas condições, pode-se provar que o conjunto de rotação vertical de $\hat{f}$ é degenerado a zero se, e somente se, $\hat{f}$ é um homeomorfismo do anel (ou seja, existem anéis invariantes em $S^{1} \times \mathbb{R}$ ), veja a figura 1.3 abaixo. Reciprocamente, se zero pertence ao conjunto de rotação vertical de $\hat{f}$ e as órbitas não são uniformemente limitadas na direção vertical, então $\hat{f}$ tem conjunto de rotação vertical com interior não vazio. E, nesse caso, veja 2.3. $f$ tem entropia topológica positiva e infinitos pontos periódicos com períodos arbitrariamente grandes.

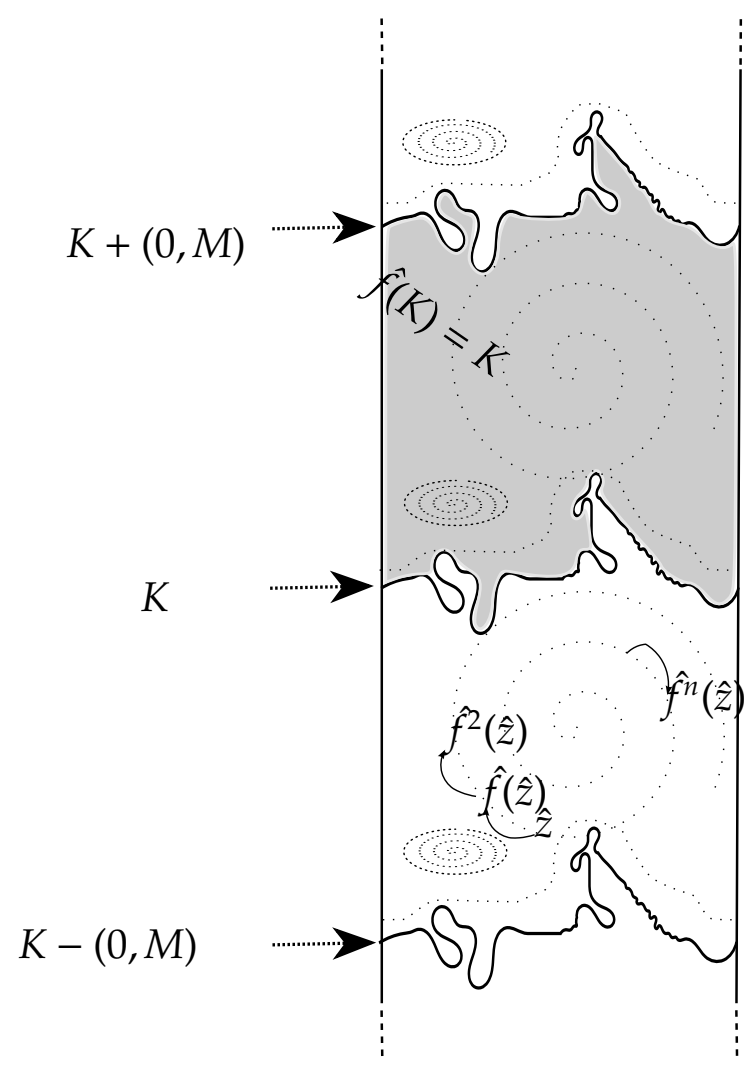

Figura 1.3: Dinâmica reduzida de $\hat{f}$ ao anel

Enunciaremos os principais resultados em duas partes: 
- Caso Conservativo

Este resultado responde positivamente a conjectura de Boyland (observe 1.2) para essa classe de homotopia.

Teorema 1.3.1. Sejam $f \in D T\left(\mathbb{T}^{2}\right)$ preservando área e um levantamento $\hat{f} \in D T\left(S^{1} \times\right.$ $\mathbb{R})$ com fluxo zero. Se $\rho_{v}(\hat{f})$ não é degenerado a zero, zero é um ponto interior de $\rho_{v}(\hat{f})$.

- Caso Geral

Teorema 1.3.2. Sejam $f \in D T\left(\mathbb{T}^{2}\right)$ e um levantamento $\hat{f} \in D T\left(S^{1} \times \mathbb{R}\right)$. Então, $\rho_{v}(\hat{f})=\{0\}$ se, e somente se, existe $K$ um continuum essencial invariante por $\hat{f}$.

Um conjunto $K \subset S^{1} \times \mathbb{R}$ é dito ser continuum se for compacto e conexo. E, é essencial se separa os fins do cilindro.

O seguinte corolário é quase imediato do teorema anterior.

Corolário 1.3.3. Sejam $f \in D T\left(\mathbb{T}^{2}\right)$ e um levantamento $\hat{f} \in D T\left(S^{1} \times \mathbb{R}\right)$ tal que $\rho_{v}(\hat{f})=[a, p / q]$ para algum racional $p / q$ e algum número real $a \leq p / q$. Então, existe $M>0$ tal que para todo ponto $\hat{z} \in S^{1} \times \mathbb{R}$,

$$
p_{2} \circ \hat{f}^{n}(\hat{z})-p_{2}(\hat{z})-n p / q<M,
$$

para todo inteiro $n>0$.

O próximo importante corolário afirma, sob certas hipóteses extremamente simples, que deslocamento sublinear vertical implica velocidade vertical. Isso é, de certo modo, uma versão mais fraca da conjectura de Boyland.

Corolário 1.3.4. Sejam $f \in D T\left(\mathbb{T}^{2}\right)$ e um levantamento $\hat{f} \in D T\left(S^{1} \times \mathbb{R}\right)$. Existe $M_{f}=M(f)>0$ tal que se para $\hat{z}_{1}, \hat{z}_{2} \in S^{1} \times \mathbb{R}$ ocorrer

$$
p_{2} \circ f^{n_{1}}\left(\hat{z}_{1}\right)-p_{2}\left(\hat{z}_{1}\right)>M_{f}
$$

$\mathrm{e}$

$$
p_{2} \circ \hat{f}^{n_{2}}\left(\hat{z}_{2}\right)-p_{2}\left(\hat{z}_{2}\right)<-M_{f}
$$

$\operatorname{com} n_{1}, n_{2} \in \mathbb{N}^{*}$, então 0 é um ponto interior de $\rho_{v}(\hat{f})$.

De fato, é possível mostrar que, sob as hipóteses anteriores, se $0 \in \rho_{v}(\hat{f})$ e, para algum ponto $\hat{z}_{1} \in S^{1} \times \mathbb{R}$,

$$
p_{2} \circ \hat{f}^{n_{1}}\left(\hat{z}_{1}\right)-p_{2}\left(\hat{z}_{1}\right)>M_{f}
$$

para algum iterado $n_{1}$, então $\rho_{v}(\hat{f})=[0, b], \operatorname{com} b>0$. 
Neste capítulo definiremos um importante invariante topológico, o conjunto de rotação, que serve para medir o deslocamento médio de um ponto no recobrimento.

\subsection{O Conjunto de Rotação}

Sejam $p: \mathbb{R}^{2} \longrightarrow \mathbb{T}^{2}$ a aplicação de recobrimento canônica do toro, definida por,

$$
p(\tilde{x}, \tilde{y})=(\tilde{x} \bmod 1, \tilde{y} \bmod 1)
$$

e $\tilde{f}: \mathbb{R}^{2} \longrightarrow \mathbb{R}^{2}$ um levantamento de um homeomorfismo do toro homotópico à identidade $f: \mathbb{T}^{2} \longrightarrow \mathbb{T}^{2}$, isto é, $\tilde{f}$ é um homeomorfismo tal que o diagrama abaixo é comutativo

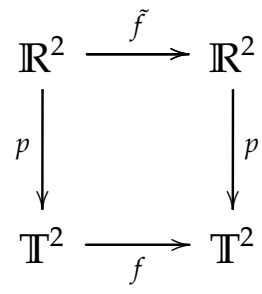

Para todo $\left(n_{1}, n_{2}\right) \in \mathbb{Z}^{2}$

$$
\tilde{f}\left((\tilde{x}, \tilde{y})+\left(n_{1}, n_{2}\right)\right)=\tilde{f}(\tilde{x}, \tilde{y})+\left(n_{1}, n_{2}\right) .
$$

Definição 2.1.1. Sejam $f: \mathbb{T}^{2} \longrightarrow \mathbb{T}^{2}$ um homeomorfismo homotópico à identidade e $\tilde{f}$ um levantamento de $f$ para o recobrimento universal. 
1. Para cada $(\tilde{x}, \tilde{y}) \in \mathbb{R}^{2}$ definimos o vetor de rotação de $(\tilde{x}, \tilde{y})$ com respeito a $\tilde{f}$ por

$$
\lim _{n \rightarrow \infty} \frac{\tilde{f}^{n}(\tilde{x}, \tilde{y})-(\tilde{x}, \tilde{y})}{n}
$$

quando o limite existir.

2. Definimos o conjunto de rotação de $\tilde{f}, \rho(\tilde{f})$, como sendo o conjunto dos pontos de acumulação do conjunto

$$
\left\{\frac{\tilde{f}^{k}(\tilde{x}, \tilde{y})-(\tilde{x}, \tilde{y})}{k}:(\tilde{x}, \tilde{y}) \in \mathbb{R}^{2}, k \in \mathbb{N}^{*}\right\} .
$$

Equivalentemente,

$$
\rho(\tilde{f})=\bigcap_{n \geq 1} f e c h o\left\{\bigcup_{k \geq n}\left\{\frac{\tilde{f}^{k}(\tilde{x}, \tilde{y})-(\tilde{x}, \tilde{y})}{k}:(\tilde{x}, \tilde{y}) \in \mathbb{R}^{2}\right\}\right\} .
$$

Assim, $v \in \rho(\tilde{f})$ se, e somente se, existem subsequências $\left\{n_{i}\right\} \subset \mathbb{N}$ e $\left\{\tilde{z}_{i}\right\} \subset \mathbb{R}^{2}$ tais que

$$
v=\lim _{i \rightarrow \infty} \frac{\tilde{f}^{n_{i}}\left(\tilde{z}_{i}\right)-\left(\tilde{z}_{i}\right)}{n_{i}} .
$$

Essa definição se deve a Misiurewicz e Ziemian [18], e definido desse modo, $\rho(\tilde{f})$ é sempre compacto e convexo (como no caso de endomorfismos do círculo [26]).

O vetor de rotação é invariante por translações de vetores de coordenadas inteiras, logo faz sentido falar no número de rotação de $(x, y) \in \mathbb{T}^{2}$. Pelo mesmo motivo, o conjunto de rotação pode ser definido usando somente pontos de um domínio fundamental, que pode ser pensado como correspondendo ao toro original.

O objetivo é usar informações sobre os vetores de rotação e o conjunto de rotação para entender a dinâmica de $f$ (deduzir a existência de pontos fixos, pontos periódicos, etc.), ver [18] e [9]. Por exemplo, foi obtido por John Franks que, se o conjunto de rotação tiver interior, então os pontos com coordenadas racionais são realizados por órbitas periódicas, isto é, para todo $\left(p_{0}, q_{0}\right) \in \mathbb{Z}^{2} \times \mathbb{N}^{*}$ (primos entre si) tal que $p_{0} / q_{0} \in \operatorname{int}\left(\rho(\tilde{f})\right.$ ), existe um ponto periódico $z \in \mathbb{T}^{2}$ (de período $q_{0}$ ) que quando levantado para o plano satisfaz, para todo $\tilde{z} \in p^{-1}(z)$,

$$
\tilde{f}^{q_{0}}(\tilde{z})=\tilde{z}+p_{0}
$$




\subsection{O Conjunto de Rotação Vertical}

Consideremos agora a classe dos homeomorfismos homotópicos à Dehn twists, $f: \mathbb{T}^{2} \longrightarrow \mathbb{T}^{2}$, isto é, tais que um levantamento de $f$ para o plano, recobrimento universal do toro, $\tilde{f}: \mathbb{R}^{2} \longrightarrow \mathbb{R}^{2}$ é um homeomorfismo que, para todo $\left(n_{1}, n_{2}\right) \in \mathbb{Z}^{2}$,

$$
\tilde{f}\left((\tilde{x}, \tilde{y})+\left(n_{1}, n_{2}\right)\right)=\tilde{f}(\tilde{x}, \tilde{y})+L_{m}\left(n_{1}, n_{2}\right),
$$

onde,

$$
L_{m}(\tilde{x}, \tilde{y})=(\tilde{x}+m \tilde{y}, \tilde{y}),
$$

é, para cada $m \in \mathbb{Z}^{*}$, uma transformação linear de $\mathbb{R}^{2}$.

A aplicação induzida no toro por $L_{m}, \phi_{L_{m}}$, é conhecida como Dehn twist. Vale observar que para cada $m \in \mathbb{Z}^{*}$ temos uma classe diferente de homotopia.

Denotemos por $D T\left(\mathbb{T}^{2}\right)$ o conjunto de homeomorfismos do toro homotópico a um Dehn twist

$$
(x, y) \mapsto \phi_{L_{m}}(x, y)=(x+m y \bmod 1, y \bmod 1),
$$

para algum $m \in \mathbb{Z}^{*}$, e seja $D T\left(\mathbb{R}^{2}\right)$ o conjunto dos levantamentos de elementos de $D T\left(\mathbb{T}^{2}\right)$ para o recobrimento universal $\mathbb{R}^{2}$.

Note que toda $L_{m}$ deixa invariante a direção horizontal do plano, permitindo definir um número de rotação (vertical) que mede a velocidade média (vertical) de um ponto $(\tilde{x}, \tilde{y})$ sobre a ação de um levantamento $\tilde{f}$. Esse fato faz com que essa classe de homotopia seja mais simples, em um certo sentido, que a da identidade. Um modo de ver isso é supor que $\tilde{f}$ tem um ponto fixo, pois nesse caso, para algum $\tilde{z} \in \mathbb{R}^{2}$,

$$
\tilde{f}(\tilde{z})=\tilde{z}
$$

e, da definição de Dehn twist,

$$
\tilde{f}(\tilde{z}+(0, k))=\tilde{z}+(0, k)+(m k, 0), \text { onde } k \in \mathbb{Z}^{*} .
$$

Sendo assim, não fica bem definido um vetor de rotação independente de $p^{-1}(p(\tilde{z}))$. Para precisar melhor considere a proposição (veja [1] e suponha $m>0$ ):

Proposição 2.2.1. Sejam $p_{1,2}: \mathbb{R}^{2} \longrightarrow \mathbb{R}$ as projeções canônicas na primeira e segunda coordenadas de $\mathbb{R}^{2}$. Sejam $f \in D T\left(\mathbb{T}^{2}\right)$ e $\tilde{f} \in D T\left(\mathbb{R}^{2}\right)$ tal que, para algum $\tilde{z} \in \mathbb{R}^{2}$, existe $C>0 \operatorname{com}\left|p_{2} \circ \tilde{f^{n}}(\tilde{z})\right|<C$, para todo $n>0$. Suponha ainda que existam $\tilde{w}_{+}$e $\tilde{w}_{-}$ tais que $p_{2} \circ \tilde{f}^{n}\left(\tilde{w}_{ \pm}\right) \longrightarrow \pm \infty$ quando $n \longrightarrow \infty$.

Então, existe $K>0$ tal que 


$$
\left|\frac{p_{1} \circ \tilde{f^{n}}(\tilde{z})-p_{1}(\tilde{z})}{n}\right|<K, \text { para todo } n>0
$$

e

$$
\frac{p_{1} \circ \tilde{f}^{n}\left(\tilde{w}_{ \pm}\right)-p_{1}\left(\tilde{w}_{ \pm}\right)}{n} \longrightarrow \pm \infty \text { quando } n \longrightarrow \infty .
$$

Assim, não faz sentido em definir um conjunto de rotação bidimensional para homeomorfismos do toro homotópico a um Dehn twist. Ao invés disso, definimos o conjunto de rotação vertical em outro recobrimento do toro, veja [3].

Seja $\pi: \mathbb{R}^{2} \longrightarrow S^{1} \times \mathbb{R}$ a aplicação de recobrimento do cilindro,

$$
\pi(\tilde{x}, \tilde{y})=(\tilde{x} \bmod 1, \tilde{y}) .
$$

Denotemos por $D T\left(S^{1} \times \mathbb{R}\right)$ o conjunto dos levantamentos de elementos de $D T\left(\mathbb{T}^{2}\right)$ para o recobrimento $S^{1} \times \mathbb{R}$. Como usado na proposição anterior, denotemos as projeções canônicas do cilindro também por $p_{1}$ e $p_{2}$ e os homeomorfismos de $D T\left(\mathbb{T}^{2}\right)$ por $f$ e seus levantamentos para o cilindro vertical e para o plano como $\hat{f}$ e $\tilde{f}$, respectivamente. A seguinte definição é dada em [2] e [7].

Definição 2.2.2. Sejam $f \in D T\left(\mathbb{T}^{2}\right)$ e um levantamento $\hat{f} \in D T\left(S^{1} \times \mathbb{R}\right)$ fixado. Para cada $\hat{z} \in S^{1} \times \mathbb{R}$ definimos o número de rotação vertical de $\hat{z}$ com respeito a $\hat{f}$, denotado por $\rho_{v}(\hat{z})$,

$$
\lim _{n \rightarrow \infty} \frac{p_{2} \circ \hat{f}^{n}(\hat{z})-p_{2}(\hat{z})}{n},
$$

quando o limite existir, onde $p_{2}: S^{1} \times \mathbb{R} \longrightarrow \mathbb{R}$ é a projeção na coordenada vertical.

Com essa definição, podemos definir o conjunto de rotação vertical, análogo ao que foi feito para a classe de homeomorfismo homotópico à identidade, digamos como o conjunto de pontos de acumulação de

$$
\left\{\frac{p_{2} \circ \hat{f}^{n}(\hat{z})-p_{2}(\hat{z})}{n}: \hat{z} \in S^{1} \times \mathbb{R}, n \in \mathbb{N}^{*}\right\} .
$$

Denotemos por $\rho_{v}(\hat{f})$ o conjunto de rotação vertical de $\hat{f}$.

Observe que a restrição para a segunda coordenada implica a importante propriedade, para todo $k \in \mathbb{Z}$,

$$
\rho_{v}(\hat{z}+(0, k))=\rho_{v}(\hat{z})
$$


onde $\hat{z} \in S^{1} \times \mathbb{R}$. Portanto, a função $\rho_{v}(\bullet)$ está também bem definida quando restrita ao toro, ou seja, depende somente de $z=p\left(\pi^{-1}(\hat{z})\right)$. Desse modo, podemos usar a notação $\rho_{v}(z)$ para isso.

Em ambos os casos, o conjunto de rotação possui as mesmas propriedades do caso unidimensional no que diz respeito a dependência do homeomorfismo no levantamento, em outras palavras:

Proposição 2.2.3. Sejam $f \in D T\left(\mathbb{T}^{2}\right)$ e um levantamento $\hat{f} \in D T\left(S^{1} \times \mathbb{R}\right)$ fixado. Então, para todo $p \in \mathbb{Z}^{*} \mathrm{e} q \in \mathbb{N}^{*}$,

1. $\rho_{v}\left(\hat{f^{q}}-(0, p)\right)=q \cdot \rho_{v}(\hat{f})-p$;

2. $\rho_{v}\left(\hat{f}^{-1}\right)=-\rho_{v}(\hat{f})$.

Para outras propriedades importantes veja [1],[7] e [8].

Vale notar que, fixado $f$ e $\hat{f}$, podemos definir a função deslocamento por

$$
\begin{aligned}
& \triangle: \mathbb{T}^{2} \longrightarrow \quad \mathbb{R} \\
& z \longmapsto \Delta(z) \doteq p_{2} \circ \hat{f}(\hat{z})-p_{2}(\hat{z}),
\end{aligned}
$$

para qualquer $\hat{z} \in \pi\left(p^{-1}(z)\right)$

Isso motiva a seguinte definição:

Definição 2.2.4. Seja $\mu$ uma medida Boreleana de probabilidade em $\mathbb{T}^{2}, f$-invariante, ou seja, $\mu \in \mathcal{M}_{\mathbb{T}^{2}}(f)$. Definimos o número de rotação vertical de $\mu$, denotada por $\rho_{v}(\mu)$, da seguinte forma

$$
\rho_{v}(\mu)=\int_{\mathbb{T}^{2}} \Delta(z) d \mu .
$$

A importância dessa definição é claramente ilustrada pelo teorema ergódico de Birkhoff, que diz que, para $\mu$-quase todo ponto $z \in \mathbb{T}^{2}$ e para qualquer $\hat{z} \in \pi\left(p^{-1}(z)\right)$,

$$
\rho_{v}(z)=\lim _{n \rightarrow \infty} \frac{1}{n} \sum_{i=0}^{n-1} \Delta \circ f^{i}(z)=\lim _{n \rightarrow \infty} \frac{p_{2} \circ \hat{f}^{n}(\hat{z})-p_{2}(\hat{z})}{n}
$$

existe e

$$
\int_{\mathbb{T}^{2}} \rho_{v}(z) d \mu=\rho_{v}(\mu)
$$


Além disso, se $f$ for ergódica com respeito a $\mu$, temos que $\rho_{v}(z)$ é constante $\mu$-quase sempre. Nesse caso, para $\mu$-quase todo ponto $z \in \mathbb{T}^{2}$ e para qualquer $\hat{z} \in \pi\left(p^{-1}(z)\right)$,

$$
\lim _{n \rightarrow \infty} \frac{p_{2} \circ \hat{f}^{n}(\hat{z})-p_{2}(\hat{z})}{n}=\rho_{v}(\mu) .
$$

Como $\rho_{v}(\bullet)$, definido em 2.4 é um funcional linear contínuo e $\mathcal{M}_{\mathbb{T}^{2}}(f)$ um conjunto convexo e compacto (na topologia fraca $\star$, ver [30]) temos que

$$
\rho_{v}\left(\mathcal{M}_{\mathbb{T}^{2}}(f)\right)=[a, b] \subset \mathbb{R}
$$

onde

$$
a=\min _{\mu \in \mathcal{M}_{\mathbb{T}^{2}}(f)} \rho_{v}(\mu) \text { e } b=\max _{\mu \in \mathcal{M}_{\mathbb{T}^{2}}(f)} \rho_{v}(\mu),
$$

ou seja, o mínimo e o máximo de $\rho_{v}(\bullet)$ quando $\mu$ varia sobre todas as medidas Boreleanas de probabilidade em $\mathbb{T}^{2}, f$-invariantes. Além disso, veja [3] e [8], para toda sequência de pontos $\hat{z}_{i} \in S^{1} \times \mathbb{R}$ e $n_{i} \longrightarrow+\infty$ (quando $i \rightarrow+\infty$ ) tal que

$$
\lim _{i \rightarrow \infty} \frac{p_{2} \circ \hat{f}^{n_{i}}\left(\hat{z}_{i}\right)-p_{2}\left(\hat{z}_{i}\right)}{n_{i}}=v,
$$

então, $v \in[a, b]$. A recíproca também é verdadeira, ou seja, $[a, b] \subset \rho_{v}(\hat{f})$. Portanto,

$$
\rho_{v}(\hat{f})=\rho_{v}\left(\mathcal{M}_{\mathbb{T}^{2}}(f)\right)=[a, b] .
$$

Definição 2.2.5. Diremos que um homeomorfismo $f$ do toro tem fluxo zero se $f$ preserva área e satisfaz $\rho_{v}(L e b)=0$ para algum levantamento fixado $\hat{f}$.

Veja a figura abaixo.

Nesse caso, se $\hat{f}$ tem fluxo zero, temos o seguinte conceito geométrico, para todo curva fechada simples homotopicamente não trivial, $\gamma: S^{1} \longrightarrow S^{1} \times \mathbb{R}$, a área da região acima de $\gamma\left(S^{1}\right)$ e abaixo de $\hat{f} \circ \gamma\left(S^{1}\right)$ é igual a área da região acima de $\hat{f} \circ \gamma\left(S^{1}\right)$ e abaixo de $\gamma\left(S^{1}\right)$. Um resultado interessante que envolve essa definição aparece em [3] e diz o seguinte, se $\hat{f}$ tem fluxo zero, então $f$ tem ponto fixo (veja também [7]). De fato, o resultado de [3] é bem mais geral:

Teorema 2.2.6. Sejam $f \in D T\left(\mathbb{T}^{2}\right)$ um levantamento $\hat{f} \in D T\left(S^{1} \times \mathbb{R}\right)$. Se $f$ não tem pontos fixos, então existe uma curva fechada simples homotopicamente não trivial que é disjunta de sua imagem por $\hat{f}$. 


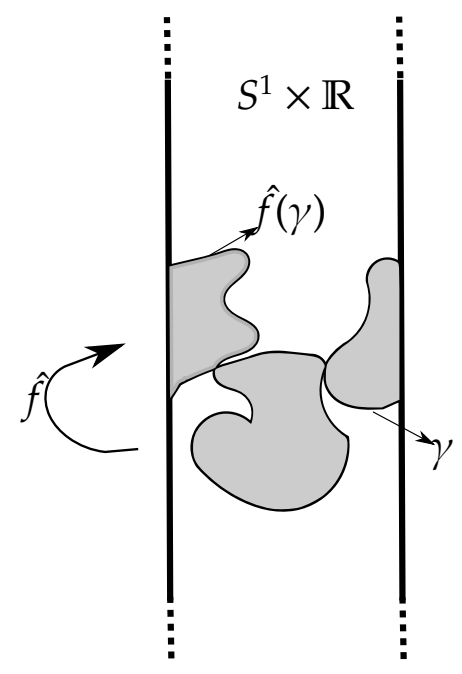

Figura 2.1: Levantamento $\hat{f}$ com fluxo zero

\section{Homotópicos à identidade Vs Dehn twists}

O estudo da geometria desses conjuntos de rotação e suas implicações dinâmicas formam uma área de pesquisa extremamente rica, atual e de grande interesse.

Um caso particularmente interessante ocorre quando estes conjuntos possuem interior vazio (no plano para homotópico à identidade e na reta para Dehn twists). Já uma das maiores dificuldades nessa teoria de rotação é saber se a existência de deslocamento sub-linear implica deslocamento linear. Isso significa, por exemplo no caso Dehn twist, que se

$$
\sup _{n \in \mathbb{N}^{*} z \in \mathbb{T}^{2}}\left\|\sum_{i=0}^{n-1} \Delta\left(f^{i}(z)\right)\right\|=\sup _{n \in \mathbb{N}^{*} z \in S^{1} \times \mathbb{R}}\left|p_{2} \circ \hat{f}^{n}(\hat{z})-p_{2}(\hat{z})\right|=+\infty,
$$

então,

$$
\rho_{v}(\hat{f}) \neq\{0\} .
$$

Isso na classe da identidade não ocorre. Em um trabalho recente de F. Tal e A. Koropecki eles apresentam o seguinte teorema.

Teorema 2.2.7. Existe um homeomorfismo do toro homotópico à identidade, $h$, que preserva área, com o conjunto de rotação contendo somente o zero e que satisfaz a propriedade de deslocamento sub-linear, ou seja, $h$ tem um levantamento para o plano, $\tilde{h}$, tal que $\tilde{h}$ tem pontos fixos e possui pontos com $\tilde{h}$-órbitas ilimitadas em qualquer direção. 
Portanto, esse exemplo mostra que a existência de deslocamento sub-linear não implica deslocamento linear, pelo menos no caso de homeomorfismos do toro homotópico à identidade. Desse modo, aplicações homotópicas a Dehn twists possuem comportamento bem diferente.

\subsection{Implicações Dinâmicas de $\rho_{v}(\bullet)$}

Apresentaremos algumas das implicações do conjunto de rotação na classe de homeomorfismos Dehn twists. Para enunciados mais precisos e demonstrações veja [7] e [2]. Na classe de homeomorfismos homotópicos à identidade veja [12].

O conjunto de rotação vertical de um homeomorfismo Dehn twist é um intervalo fechado (possivelmente um ponto, mas nunca vazio).

O próximo resultado afirma que se um homeomorfismo Dehn twist tem conjunto de rotação vertical com interior não vazio, então esse possui complexidade dinâmica "maior que" a de um pseudo-Anosov (veja [11] para algumas importantes propriedades dinâmicas de um pseudo-Anosov).

Teorema 2.3.1. Sejam $f \in D T\left(\mathbb{T}^{2}\right)$ e um levantamento $\hat{f} \in D T\left(S^{1} \times \mathbb{R}\right)$ tal que $\rho_{v}(\hat{f})$ tenha interior não vazio. Então, $f$ é isotópico a uma aplicação pseudo-Anosov relativo a um conjunto finito invariante. Consequentemente, $f$ tem entropia topológica positiva.

Muito mais pode ser dito se $\rho_{v}(\hat{f})=[a, b] \operatorname{com} a<b$. De fato,

- para todo $w \in] a, b\left[\right.$ existe um compacto, $f$-invariante $Q_{w}$ com

$$
\rho_{v}(z)=\lim _{n \rightarrow+\infty} \frac{1}{n} \sum_{0 \leq i \leq n-1} \Delta\left(f^{i}(z)\right)=w
$$

para todo $z \in Q_{w}$. Se $w=p / q$ é um número racional, $Q_{\frac{p}{q}}$ é uma órbita periódica.

- os extremos de $\rho_{v}(\hat{f})$ são realizados por medidas de probabilidade ergódicas, isto é, existem medidas $\mu_{a}$ e $\mu_{b}$ ergódicas tais que $\rho_{v}\left(\mu_{a}\right)=a$ e $\rho_{v}\left(\mu_{b}\right)=b$. Assim, pelo teorema ergódico de Birkhoff, existem pontos $z_{a}$ e $z_{b}$ tais que $\rho_{v}\left(z_{a}\right)=$ $a$ e $\rho_{v}\left(z_{b}\right)=b$.

- para toda $\mu \in \mathcal{M}_{\mathbb{T}^{2}}(f)$ temos $\rho_{v}(\mu) \in[a, b]$. 
- em geral, não é verdade que os pontos extremais racionais do conjunto de rotação vertical são realizados por orbitas periódicas (isso no caso homotópico à identidade é falso).

A figura abaixo exemplifica a notação que iremos adotar ao longo do trabalho. 


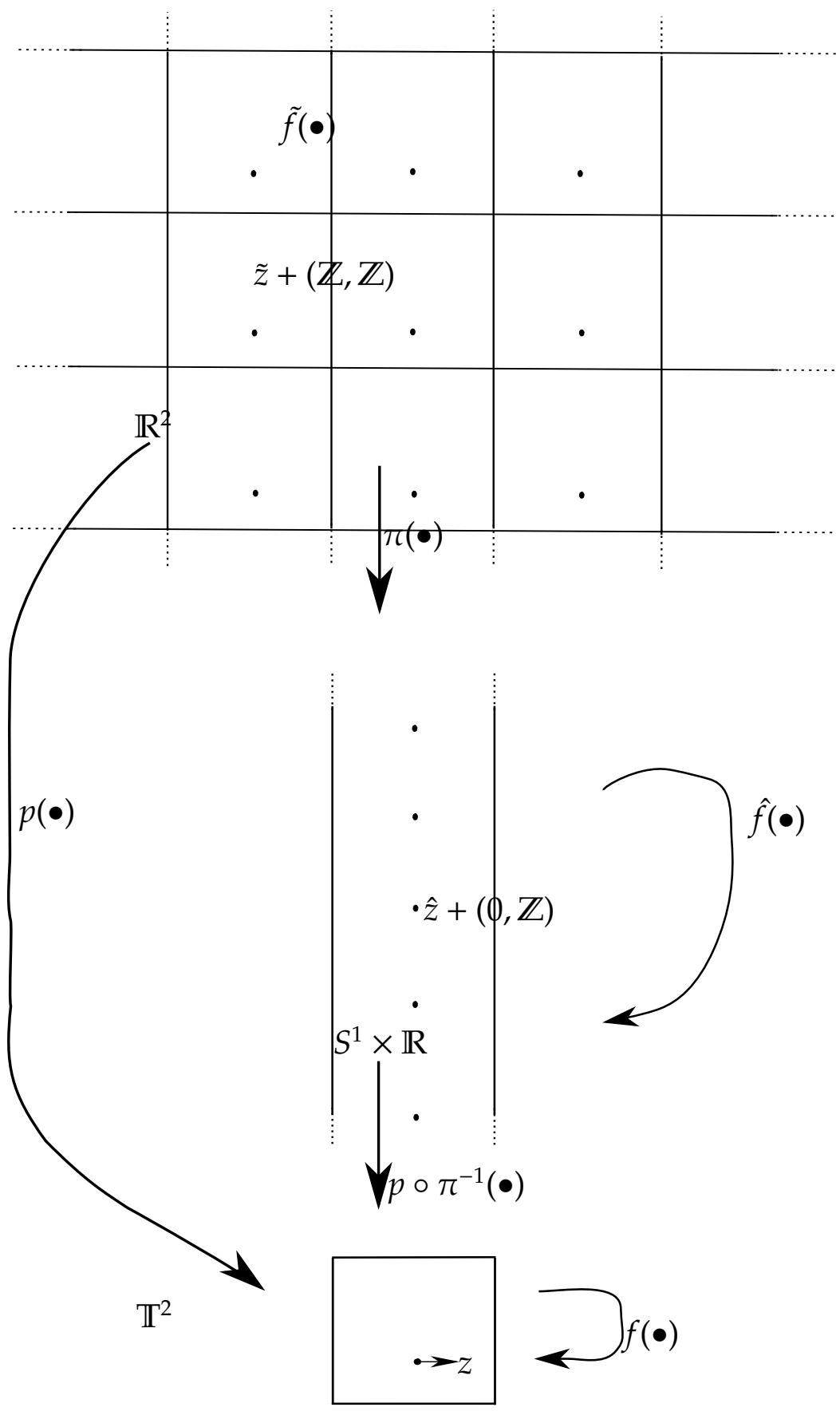

Figura 2.2: Aplicações de recobrimento. 


\section{CAPÍTULO 3}

\section{FERRAMENTAS BÁSICAS}

Nessa seção apresentaremos a teoria desenvolvida em [5] e [4]. Basicamente, a técnica é compactificar a dinâmica no recobrimento e estudar a dinâmica induzida nas vizinhanças dos pontos no infinito.

\subsection{Os conjuntos $B_{S}^{-}$e $B_{N}^{+}$}

Para estendermos as construções feita em [5] e [4] para nosso contexto, consideremos $f \in D T\left(\mathbb{T}^{2}\right)$, um levantamento $\hat{f} \in D T\left(S^{1} \times \mathbb{R}\right)$ e um levantamento de $\hat{f}$ para o plano, denotado por $\tilde{f}$.

Definição 3.1.1. Para cada número real $a$, definamos os conjuntos

$$
\left.\left.H_{a}=S^{1} \times\{a\}, H_{a}^{-}=S^{1} \times\right]-\infty, a\right] \text { e } H_{a}^{+}=S^{1} \times[a,+\infty[.
$$

Quando $a=0$ escreveremos simplesmente $H, H^{-}$e $H^{+}$.

Consideremos a compactificação do cilindro vertical, $S^{1} \times \mathbb{R}$, denotada por $N$, $S$-compactificação, isto é, adicionamos dois pontos $N$ (fim superior) e $S$ (fim inferior) ao cilindro, obtendo a esfera topológica $S^{2}$. Seja $\bar{f}: S^{2} \longrightarrow S^{2}$ o homeomorfismo induzido por $\hat{f}: S^{1} \times \mathbb{R} \longrightarrow S^{1} \times \mathbb{R}$. Então, $\bar{f}(N)=N$ e $\bar{f}(S)=S$ (veja a figura 3.1 abaixo). 


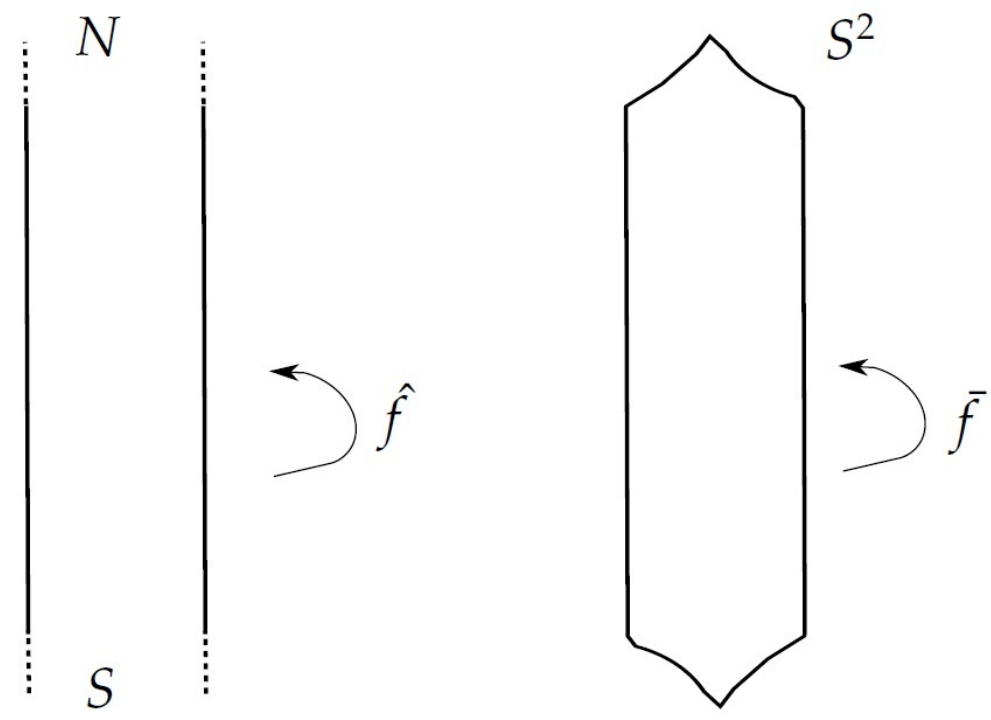

Figura 3.1: Dinâmica na compactificação

Denotemos por $\bar{A}$, um conjunto de $S^{2}$, se esse for correspondente do conjunto $A$ no cilindro. Nessas condições, temos que os conjuntos fechados

$$
\bar{B}^{-}=\bigcap_{n \leq 0} \bar{f}^{n}\left(\bar{H}^{-}\right)
$$

$\mathrm{e}$

$$
\bar{B}^{+}=\bigcap_{n \leq 0} \bar{f}^{n}\left(\bar{H}^{+}\right),
$$

são $\bar{f}$-positivamente invariantes com $S \in \bar{B}^{-}$e $N \in \bar{B}^{+}$. De fato, são fechados (e nunca vazios) pois são interseções de conjuntos fechados da forma $\bar{f}^{-n}\left(\bar{H}^{ \pm}\right)$, com $n \geq 0$, que contém um dos pontos fixos $N$, S. Pelo cálculo direto de $\bar{f}\left(\bar{B}^{ \pm}\right)$, $\bar{f}\left(\bar{H}^{ \pm} \cap \bar{f}^{-1}\left(\bar{H}^{ \pm}\right) \cap \bar{f}^{-2}\left(\bar{H}^{ \pm}\right) \cap \ldots\right)=\bar{f}\left(\bar{H}^{ \pm}\right) \cap \bar{H}^{ \pm} \cap \bar{f}^{-1}\left(\bar{H}^{ \pm}\right) \cap \bar{f}^{-2}\left(\bar{H}^{ \pm}\right) \cap \ldots=\bar{f}\left(\bar{H}^{ \pm}\right) \bigcap \bar{B}^{ \pm} \subset \bar{B}^{ \pm}$ temos que são positivamente invariantes.

Os conjuntos $\bar{B}_{S}^{-}$e $\bar{B}_{N}^{+}$são, respectivamente, as componentes conexas de $\bar{B}^{-}$e $\bar{B}^{+}$que contém $S$ e $N$. Desse modo, $B_{S}^{-}$e $B_{N}^{+}$são os correspondentes conjuntos do 
cilindro. Em outras palavras,

$$
B_{S}^{-}=\text {União das componentes conexas ilimitadas de } B^{-}=\bigcap_{n \leq 0} \hat{f}^{n}\left(H^{-}\right)
$$

e

$$
B_{N}^{+}=\text {União das componentes conexas ilimitadas de } B^{+}=\bigcap_{n \leq 0} \hat{f}^{n}\left(H^{+}\right) \text {. }
$$

Assim, esses conjuntos são sempre fechados e $\hat{f}\left(B_{S}^{-}\right) \subset B_{S^{\prime}}^{-}, \hat{f}\left(B_{N}^{+}\right) \subset B_{N^{\prime}}^{+}$mas eventualmente vazios. Provaremos que, se o zero pertence ao conjunto de rotação vertical de $\hat{f}$, então $B_{S}^{-}$e $B_{N}^{+}$são não vazios.

Antes de enunciarmos precisamente esse teorema, consideremos os seguintes lemas intermediários:

Lema 3.1.1. Suponha que $0 \in \rho_{v}(\hat{f})$ e que para um dado $M>0$ existem um inteiro positivo $n$ e um ponto $\hat{z} \in S^{1} \times[0,1]$ tal que $p_{2} \circ \hat{f}^{n}(\hat{z})>M$. Então $B_{N}^{+} \cap H \neq \emptyset$ (equivalentemente para $S^{2}, \bar{B}_{N}^{+} \cap \bar{H} \neq \emptyset$ ).

Analogamente temos:

Lema 3.1.2. Suponha que $0 \in \rho_{v}(\hat{f})$ e que para um dado $M>0$ existem um inteiro positivo $n$ e um ponto $\hat{z} \in S^{1} \times[0,1]$ tal que $p_{2} \circ \hat{f}^{n}(\hat{z})<-M$. Então $B_{S}^{-} \cap H \neq \emptyset$ (equivalentemente para $S^{2}, \bar{B}_{S}^{-} \cap \bar{H} \neq \emptyset$ ).

A prova desses dois lemas é análoga e aparece em um contexto diferente em Le Calvez [13] e em Birkhoff [22]. Portanto, demonstraremos somente o último.

Demonstração. Primeiramente, observe que se para algum inteiro $M>0$, existir um inteiro positivo $n_{0}$ tal que $\hat{f}^{n_{0}}\left(H^{-}\right)$está contido em $H_{-M}^{-}=H^{-}-(0, M)$, ou seja,

$$
\hat{f}^{n_{0}}\left(H^{-}\right) \subset H_{-M}^{-}
$$

Aplicando $\hat{f}^{n_{0}} \mathrm{em} 3.3$.

$$
f^{2 n_{0}}\left(H^{-}\right) \subset \hat{f}^{n_{0}}\left(H^{-}-(0, M)\right)=\hat{f}^{n_{0}}\left(H^{-}\right)-(0, M),
$$

$\operatorname{logo} f^{2 n_{0}}\left(H^{-}\right) \subset H^{-}-(0,2 M)$. Indutivamente, $k \in \mathbb{N}^{*}$,

$$
f^{k n_{0}}\left(H^{-}\right) \subset H^{-}-(0, k M) .
$$


Assim, para todo ponto $\hat{z} \in H^{-}, p_{2} \circ f^{k n_{0}}(\hat{z}) \leq-k M$. Mas, como $H^{-}$contém um domínio fundamental, obtemos que para todo ponto $\hat{z} \in S^{1} \times \mathbb{R}$,

$$
p_{2} \circ f^{k n_{0}}(\hat{z})-p_{2}(\hat{z}) \leq-k M .
$$

Nessas condições,

$$
p_{2} \circ f^{k n_{0}}(\hat{z})-p_{2}(\hat{z}) \leq-k M
$$

para todo ponto do cilindro e para todo $k$ inteiro positivo. Portanto,

$$
\limsup _{i \rightarrow \infty} \frac{p_{2} \circ \hat{f}^{i}(\hat{z})-p_{2}(\hat{z})}{i} \leq \frac{-M}{n_{0}}<0
$$

para todo $\hat{z} \in S^{1} \times \mathbb{R}$. O que é um absurdo, pois estamos supondo que $0 \in \rho_{v}(\hat{f})$.

Sendo assim, as hipóteses do lema implicam que para todo $M>0$ existe um inteiro positivo $n=n(M)$, tal que

$$
f^{n}(H) \bigcap H_{-M} \neq \emptyset .
$$

Claramente, $M$ e $n$ tem a seguinte relação: tomando $M$ cada vez maior, necessitamos de $n(M)$ também maior para que $\hat{f}^{n(M)}(H) \cap H_{-M}^{-} \neq \emptyset$.

Desse modo, dado $N>0$ existe $a \leq-2$ tal que

$$
n=\inf \left\{i>0: \hat{f}^{i}(H) \cap H_{a} \neq \emptyset\right\}>N .
$$

Além disso, por definição de $n, \hat{f^{i}}(H) \subset H_{a}^{+}$para todo $i \in\{0,1, \ldots, n-2, n-1\}$. Equivalentemente,

$$
\hat{f}^{-i}\left(H_{a}\right) \subset H^{-} \quad \forall i \in\{0,1, \ldots, n-2, n-1\}
$$

e

$$
\hat{f}^{-n}\left(H_{a}\right) \cap H \neq \emptyset .
$$

Assim, existe um arco contínuo simples

$$
\Gamma_{N} \subset \hat{f}^{-n}\left(H_{a}^{-}\right) \cap H^{-}
$$

tal que $\bar{\Gamma}_{N}$ conecta $S$ a $\bar{H}$ (um fim de $\bar{\Gamma}_{N}$ é $S$ e o outro está em $\bar{H}$ ). Veja a figura 3.2 abaixo.

Portanto, como $\Gamma_{N} \subset \hat{f}^{-n}\left(H_{a}^{-}\right) \cap H^{-}$,

$$
\hat{f}^{\hat{i}}\left(\Gamma_{N}\right) \subset \hat{f}^{-n+i}\left(H_{a}^{-}\right) \subset H^{-} \quad \forall i \in\{0,1, \ldots, n-1, n\} .
$$


Em outras palavras,

$$
\Gamma_{N} \subset \bigcap_{i=0}^{n} \hat{f}^{-i}\left(H^{-}\right)
$$
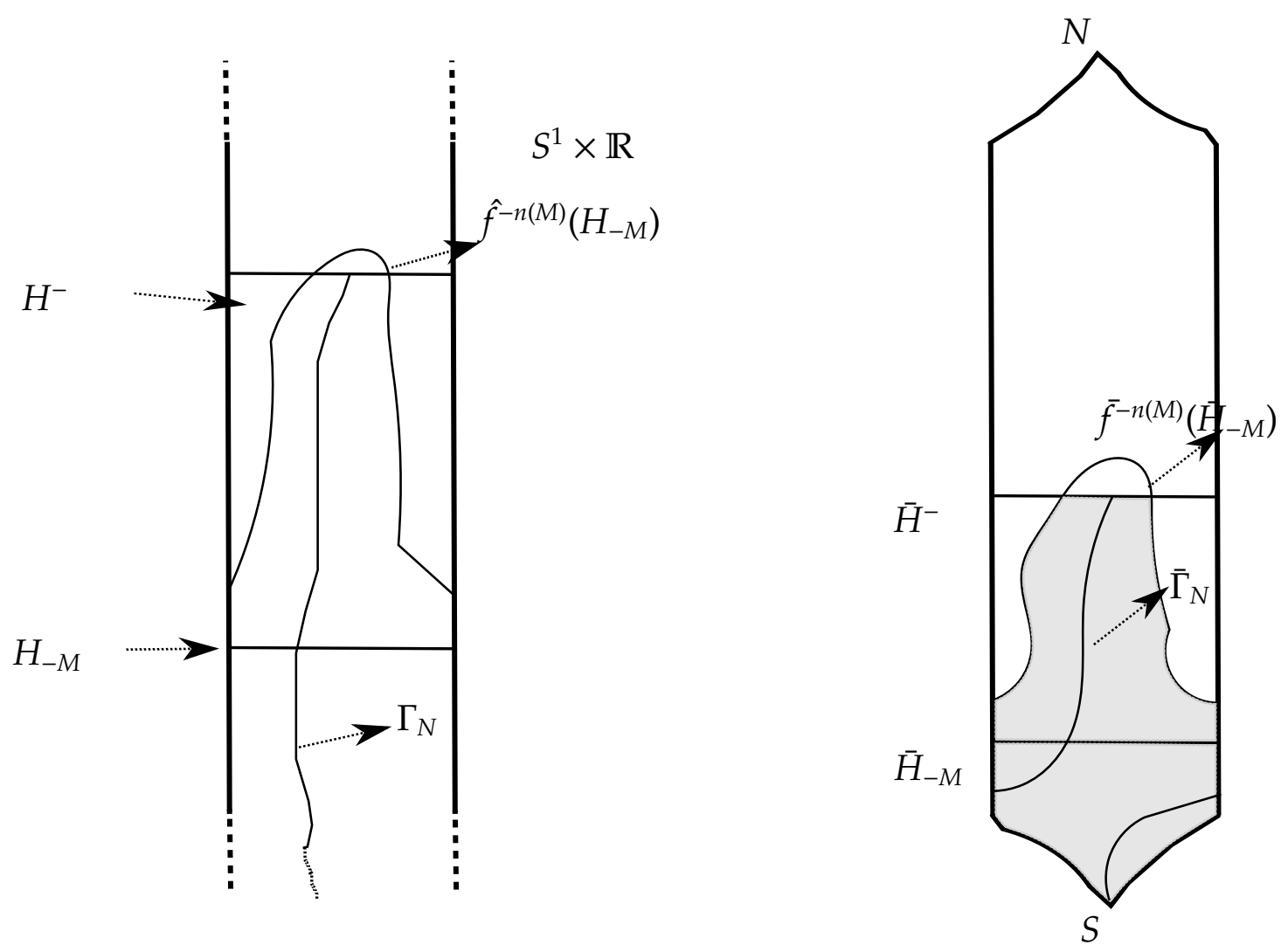

$S^{2}$

Figura 3.2: Arcos contínuos simples $\Gamma_{N}$ e $\bar{\Gamma}_{N}$

Na compactificação $S^{2}$, temos uma sequência $\left\{\bar{\Gamma}_{N}\right\}_{N>0}$ de conjuntos compactos conexos, logo existe um conjunto $\bar{\Gamma}$ compacto, conexo e que conecta $S$ a $H$, onde $\bar{\Gamma}$ é o limite de uma subsequência convergente na topologia de Hausdorff de $\left\{\bar{\Gamma}_{N}\right\}_{N>0}$, isto é, $\bar{\Gamma}_{N_{i}} \rightarrow \bar{\Gamma}$ quando $i \rightarrow \infty$. Assim, dado $\epsilon>0$, se $V_{\epsilon}(\bar{\Gamma})$ e $V_{\epsilon}\left(\bar{\Gamma}_{N_{i}}\right)$ são $\epsilon$-vizinhanças de $\bar{\Gamma}$ e de $\bar{\Gamma}_{N_{i}}$, existe $k>0$ tal que

$$
\forall N_{i} \geq k \quad \bar{\Gamma}_{N_{i}} \subset V_{\epsilon}(\bar{\Gamma}) \text { e } \bar{\Gamma} \subset V_{\epsilon}\left(\bar{\Gamma}_{N_{i}}\right)
$$


donde segue as propriedades de $\bar{\Gamma}$. Por construção, $N \longrightarrow+\infty$ implica que $n \longrightarrow+\infty$ e como $\bar{\Gamma}_{N} \subset \bigcap_{i=0}^{n} \bar{f}^{-i}\left(\bar{H}^{-}\right)$temos que $\bar{\Gamma} \subset \bigcap_{i=0}^{\infty} \bar{f}^{-i}\left(\bar{H}^{-}\right)=\bar{B}_{S}^{-}$. Isso conclui o lema.
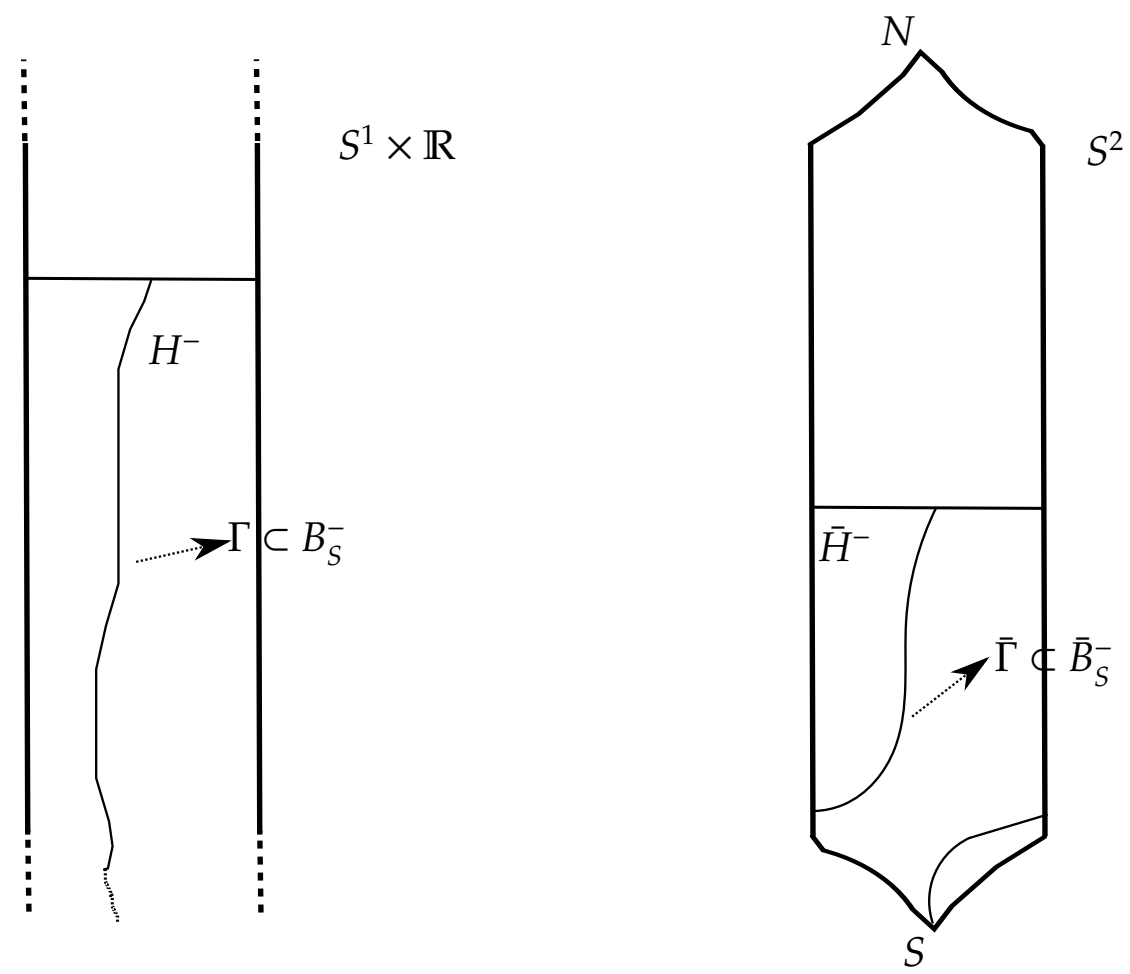

Figura 3.3: $B_{S}^{-}$e $\bar{B}_{S}^{-}$não vazios.

Esses lemas garantem que sob a hipótese extra de pontos com deslocamentos ilimitados em ambas as direções, isto é, fecho $\left(\bigcup_{n \geq 1} \bar{f}^{n}(\bar{H})\right) \supset\{S, N\}$ implica que $B_{S}^{-}$e $B_{N}^{+}$são não vazios. Mas, como dito anteriormente, a existência desses conjuntos é mais geral.

Teorema 3.1.2. Se $0 \in \rho_{v}(\hat{f})$, então $B_{S}^{-} \neq \emptyset$ e $B_{N}^{+} \neq \emptyset$. 

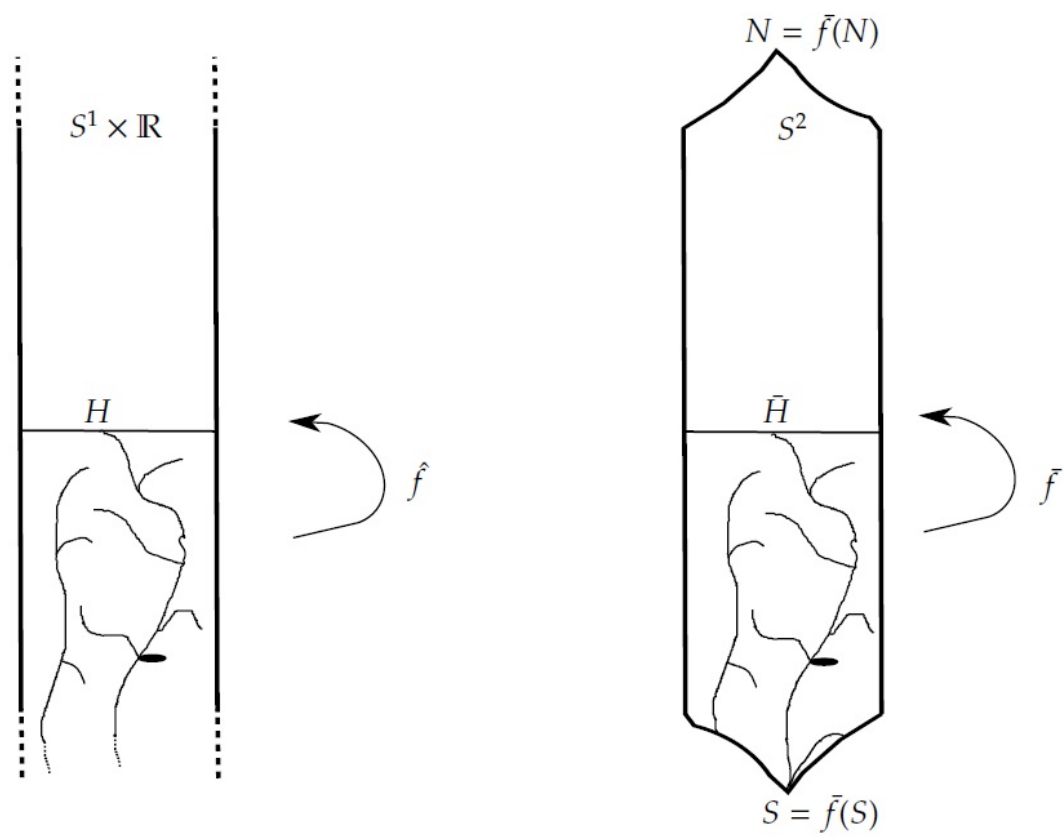

Figura 3.4: Os conjuntos $B_{S}^{-}$e $\bar{B}_{S}^{-}$

Demonstração. Suponha que para cada $M>1$, existem inteiros positivos $n^{-}$e $n^{+}$e existem pontos $\hat{z}^{-}, \hat{z}^{+} \in S^{1} \times[0,1]$ tais que

$$
p_{2} \circ \hat{f}^{n^{-}}\left(\hat{z}^{-}\right)-p_{2}\left(\hat{z}^{-}\right)<-M \text { e } p_{2} \circ \hat{f}^{n^{+}}\left(\hat{z}^{+}\right)-p_{2}\left(\hat{z}^{+}\right)>M .
$$

Nesse caso, o lema 3.1.1 e o lema 3.1.2 implicam que $B_{S}^{-}$e $B_{N}^{+}$são não vazios.

Suponha que a órbita de todo ponto seja uniformemente limitada no cilindro, isto é, existe um inteiro $M_{0}>0$ tal que

$$
\bigcup_{n \in \mathbb{Z}} \hat{f}^{n}\left(S^{1} \times[0,1]\right) \subset S^{1} \times\left[-M_{0}, M_{0}\right] .
$$

Considere o conjunto

$$
O=\bigcup_{n \in \mathbb{Z}} \hat{f}^{n}\left(S^{1} \times\right]-\infty, 0[)
$$

Assim, $O$ é aberto e invariante,

$$
\hat{f}(O)=\hat{f}\left(\bigcup_{n \in \mathbb{Z}} \hat{f}^{n}\left(S^{1} \times\right]-\infty, 0[)=\bigcup_{n \in \mathbb{Z}} \hat{f}^{n}\left(S^{1} \times\right]-\infty, 0[=0 .\right.
$$

Por 3.4 temos que o bordo de $O$ é um subconjunto de $\left.S^{1} \times\right]-\infty, M_{0}$ ] e existe $K$, a única componente de $\partial O$ conexa, que separa os fins do cilindro e tal que $K \subset S^{1} \times\left[-M_{0}, M_{0}\right]$. 
Sendo $O$ invariante por $\hat{f}, \hat{f}(K)=K$.

Portanto, Ké um compacto, conexo que separa os fins do cilindro $\hat{f}$-invariante. Desse modo, existe um transladado inteiro de $K$ que está abaixo de $H$, ou seja, existe (o primeiro) $M_{1} \geq 0$ tal que

$$
K-\left(0, M_{1}\right) \subset H^{-} .
$$

Sendo $K-\left(0, M_{1}\right) \hat{f}$-invariante, temos que a componente conexa do complementar de $K-\left(0, M_{1}\right)$ que contém o fim inferior do cilindro também é $\hat{f}$-invariante e, portanto, está contida em $B_{S}^{-}$.

De um modo análogo, obtemos que $B_{N}^{+}$é não vazio.

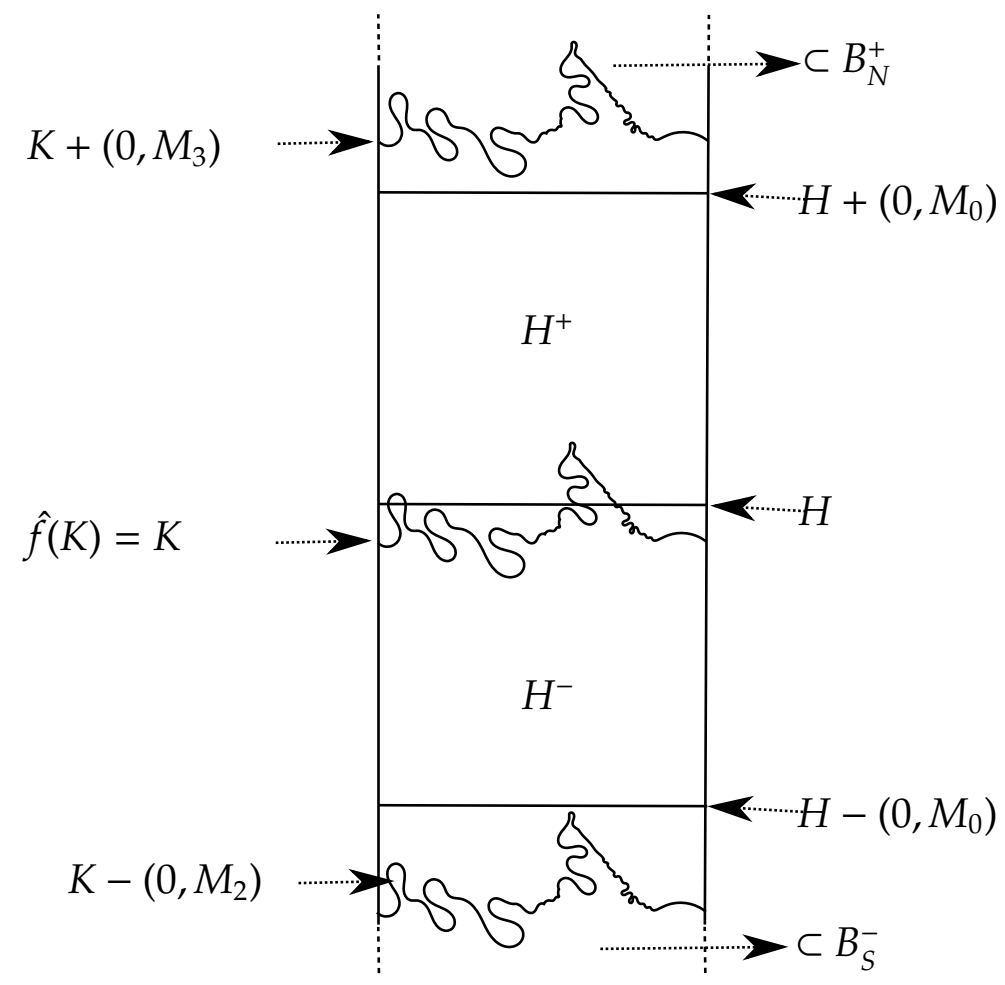

Figura 3.5: Órbitas uniformemente limitadas no cilindro 
No próximo caso, vamos supor que existem órbitas ilimitadas em apenas uma direção. Em outras palavras, existe $M_{0}>0$ tal que

$$
\bigcup_{n>0} \hat{f}^{n}\left(S^{1} \times[0,1]\right) \subset S^{1} \times\left[-M_{0},+\infty[,\right.
$$

então existe um conjunto compacto conexo $K \subset S^{1} \times \mathbb{R}$, tal que $K$ separa os fins do cilindro e $K$ é o bordo do conjunto aberto e conexo $O=\bigcup_{n \geq 0} \hat{f}^{n}\left(S^{1} \times\right] 0,+\infty[)$. Note que $O$ contém o fim superior do cilindro e $\hat{f}(O) \subset O$.

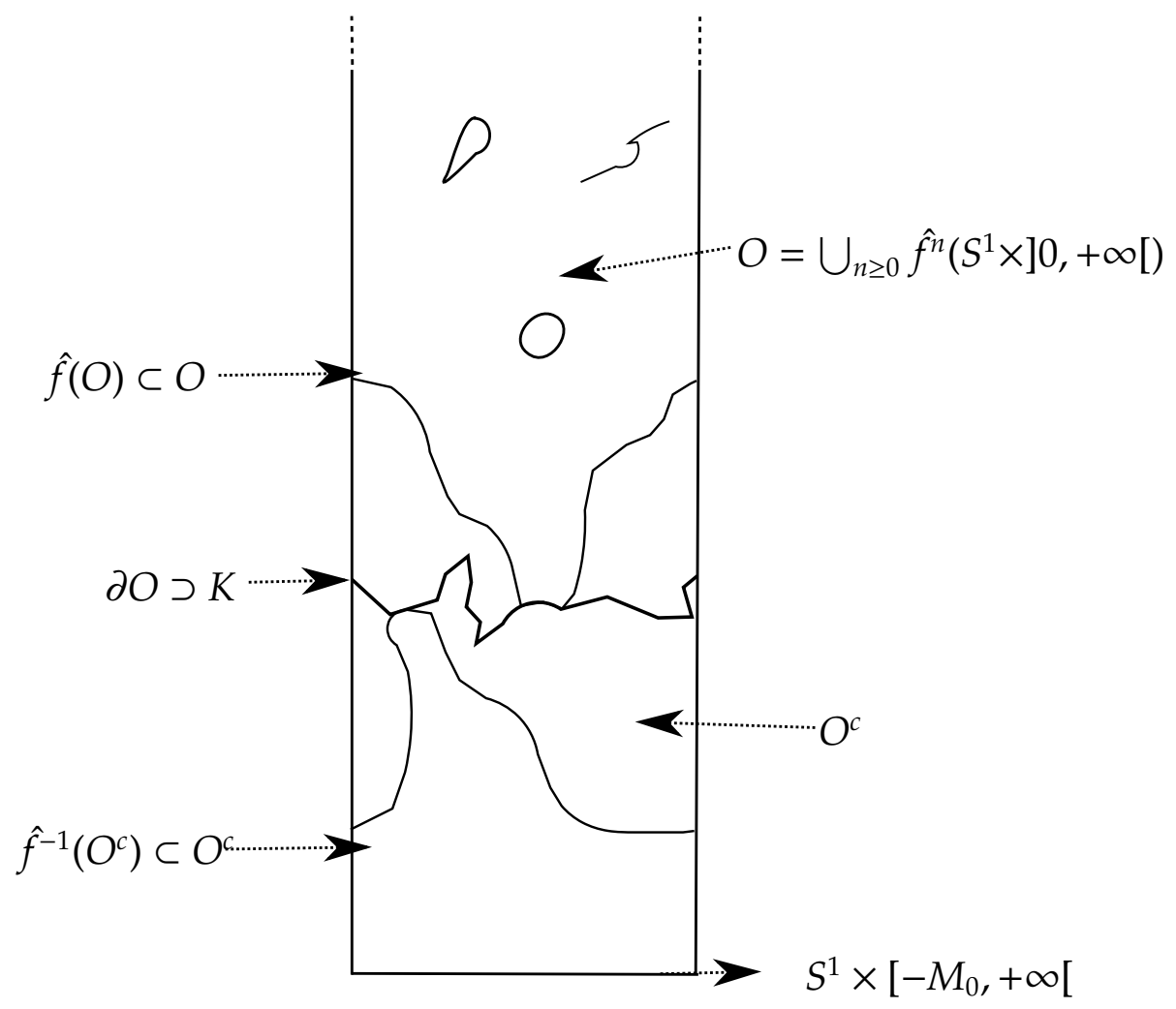

Figura 3.6: Órbitas ilimitadas em apenas uma direção do cilindro

Se

$$
\bigcap_{n \geq 0} \hat{f}^{n}(f \operatorname{ech} o(O))=\emptyset
$$


então $0 \notin \rho_{v}(\hat{f})$, pois, neste caso existiria um inteiro $N_{1}>1$ tal que $\hat{f}^{N_{1}}(H) \subset H^{+}+(0,1)$. Assim,

$$
\bigcap_{n \geq 0} \hat{f}^{n}(\operatorname{fecho}(O))
$$

é um subconjunto de $O$ fechado, conexo, ilimitado e $\hat{f}$-invariante. Portanto, $B_{N}^{+}$é não vazio.

Como $\hat{f}(O) \subset O, \hat{f}\left(O^{c}\right) \supset O^{c}$, ou seja, $\hat{f}^{-1}\left(O^{c}\right) \subset O^{c}$, desse modo, fazendo a mesma construção para $O^{c}$, obtemos que

$$
\bigcap_{n \leq 0} \hat{f}^{n}\left(O^{c}\right)
$$

é um subconjunto de $O^{c}$ fechado, conexo, ilimitado e $\hat{f}$-invariante. Donde vem que $B_{S}^{-}$é não vazio.

A última possibilidade pode ser tratada de um modo análogo.

\subsection{Os conjuntos $\omega$-limite de $B_{S}^{-}$e $B_{N^{*}}^{+}$.}

Nessa subseção examinaremos algumas propriedades do conjunto $\omega$-limite de $\bar{B}_{S}^{-}$, denotado por $\omega\left(\bar{B}_{S}^{-}\right)$, e definido como segue:

$$
\omega\left(\bar{B}_{S}^{-}\right)=\bigcap_{n \geq 0} \text { fecho }\left\{\bigcup_{i \geq n} \bar{f}^{\bar{\imath}}\left(\bar{B}_{S}^{-}\right)\right\} .
$$

Como $\bar{f}\left(\bar{B}_{S}^{-}\right) \subset \bar{B}_{S}^{-}$e $\bar{B}_{S}^{-}$é fechado, temos

$$
\omega\left(\bar{B}_{S}^{-}\right)=\bigcap_{n=0}^{\infty} \bar{f}^{n}\left(\bar{B}_{S}^{-}\right) .
$$

Nessas condições, $\omega\left(\bar{B}_{S}^{-}\right)$é a intersecção de uma sequência encaixante de compactos conexos e, portanto, é um conjunto compacto conexo (possivelmente um único ponto, mas nunca vazio). Veremos que, se o omega limite de $\bar{B}_{S}^{-}$é, somente, o ponto $S, \bar{B}_{S}^{-}$pode ser pensado como sendo o conjunto estável de $S$. Decorre da definição 3.5 acima o seguinte lema:

Lema 3.2.1. $\omega\left(B_{S}^{-}\right)$é um conjunto fechado, $\hat{f}$-invariante, limitado superiormente (de fato $\left.\omega\left(B_{S}^{-}\right) \subset B^{-} \subset H^{-}\right)$e com todas as suas componentes conexas ilimitadas. 
Demonstração. As propriedades fechado, $\hat{f}$-invariante e limitado superiormente são consequências imediatas da definição do conjunto. Com efeito, como $\hat{f}^{n}\left(B_{S}^{-}\right)$é fechado para todo $n \in \mathbb{N}$ e $\hat{f}\left(B_{S}^{-}\right) \subset B_{S}^{-} \subset H^{-}$temos que $\omega\left(B_{S}^{-}\right)=\bigcap_{n=0}^{\infty} \hat{f}^{n}\left(B_{S}^{-}\right)$é um subconjunto fechado de $\mathrm{H}^{-}$. O seguinte cálculo fornece a prova da invariância por $\hat{f}$. Sendo que $\hat{f}\left(B_{S}^{-}\right) \subset B_{S}^{-}$,

$$
\hat{f}\left(\omega\left(B_{S}^{-}\right)\right)=\hat{f}\left(\cap_{n=0}^{\infty} \hat{f}^{n}\left(B_{S}^{-}\right)\right)=\cap_{n=1}^{\infty} \hat{f}^{n}\left(B_{S}^{-}\right)=\cap_{n=1}^{\infty} \hat{f}^{n}\left(B_{S}^{-}\right) \bigcap B_{S}^{-}=\omega\left(B_{S}^{-}\right) .
$$

Como $S \in \bar{B}_{S}^{-}$e $\bar{f}(S)=S$, obtemos que $S \in \omega\left(\bar{B}_{S}^{-}\right)$. Assim, tendo que $\omega\left(\bar{B}_{S}^{-}\right)$é conexo, cada componente conexa de $\omega\left(B_{S}^{-}\right)$é ilimitada.

Note que, como $B_{S}^{-}$é fechado e positivamente invariante por $\hat{f}, \omega\left(B_{S}^{-}\right) \subset B_{S}^{-}$. Mas ainda é possível que $\omega\left(B_{S}^{-}\right)$seja vazio. O próximo lema assegura que se esse é o caso então o conjunto de rotação vertical de $\hat{f}$ não será degenerado, ou seja, $\rho_{v}(\hat{f})=[a, b]$ $\operatorname{com} a<b$.

Lema 3.2.2. Suponha que $0 \in \rho_{v}(\hat{f})$ (então, $B_{S}^{-}$é não vazio) e que $\omega\left(B_{S}^{-}\right)=\emptyset$. Então, $\rho_{v}(\hat{f}) \supset[-\epsilon, 0]$, para algum $\epsilon>0$.

Demonstração. Como $0 \in \rho_{v}(\hat{f})$, temos, pelo teorema 3.1.2. que $B_{S}^{-}$é não vazio. Por definição, $\hat{f}\left(B_{S}^{-}\right) \subset B_{S}^{-}$e $\omega\left(B_{S}^{-}\right)=\cap_{n=0}^{\infty} \hat{f}^{n}\left(B_{S}^{-}\right)$. Por hipótese $\omega\left(B_{S}^{-}\right)$é vazio.

Desse modo, se para todo inteiro $N \geq 0$ existir um inteiro $n \geq N$ tal que

$$
\hat{f}^{n}\left(B_{S}^{-}\right) \bigcap H-(0,1) \neq \emptyset,
$$

teríamos que

$$
\forall k \in\{0,1, \ldots, n\}, \hat{f}^{k}\left(B_{S}^{-}\right) \bigcap H-(0,1) \neq \emptyset,
$$

pois, $\hat{f}^{n}\left(B_{S}^{-}\right) \subset \hat{f}^{k}\left(B_{S}^{-}\right), \quad \forall k \in\{0,1, \ldots, n\}$.

Mas isso implica o absurdo, $\bigcap_{n=0}^{\infty} \hat{f}^{n}\left(B_{S}^{-}\right) \bigcap H-(0,1) \neq \emptyset$.

Portanto, existe um inteiro $N_{1}>0$ tal que para todo $n \geq N_{1}$,

$$
\hat{f}^{n}\left(B_{S}^{-}\right) \subset H^{-}-(0,1) .
$$

De fato, podemos provar que

$$
\hat{f}^{N_{1}}\left(B_{S}^{-}\right) \subset B_{S}^{-}-(0,1) .
$$


Suponha, por contradição, que

$$
\hat{f}^{N_{1}}\left(B_{S}^{-}\right) \not \subset B_{S}^{-}-(0,1) .
$$

Assim, existe um ponto $\hat{z} \in B_{S}^{-}$tal que

$$
\hat{f}^{N_{1}}(\hat{z}) \notin B_{S}^{-}-(0,1) \text {. }
$$

Seja $\gamma$ a componente conexa de $B_{S}^{-}$que contém o ponto $\hat{z}$. Então, por 3.6. $\hat{f}^{N_{1}}(\gamma) \subset H_{-1}^{-}$ e $\hat{f}^{N_{1}}(\gamma) \not \subset B_{S}^{-}-(0,1)$, ou seja,

$$
\hat{f}^{N_{1}}(\gamma)+(0,1) \not \subset B_{S}^{-} .
$$

Como $\hat{f}^{N_{1}}(\gamma)+(0,1)$ é um subconjunto de $H^{-}$conexo e ilimitado, deve existir um inteiro $\mathrm{N}_{2}>0$ tal que

$$
\hat{f}^{N_{2}}\left(\hat{f}^{N_{1}}(\gamma)+(0,1)\right) \not \subset H^{-},
$$

pois vale 3.7. Equivalentemente,

$$
\hat{f}^{N_{1}+N_{2}}(\gamma)+(0,1) \not \subset H^{-},
$$

isto é, $\hat{f}^{N_{1}+N_{2}}(\gamma) \not \subset H^{-}-(0,1)$. O que é um absurdo, já que vale 3.6 . Nessas condições, $\hat{f}^{N_{1}}\left(B_{S}^{-}\right) \subset B_{S}^{-}-(0,1)$, para algum $N_{1}>0$.

Sendo assim, indutivamente em $k \in \mathbb{N}^{*}, f^{k N_{1}}\left(B_{S}^{-}\right) \subset B_{S}^{-}-(0, k) \subset H^{-}-(0, k)$, donde segue, para todo $\hat{z} \in B_{S}^{-}$,

$$
\limsup _{n \rightarrow+\infty} \frac{p_{2}\left(\hat{f}^{n}(\hat{z})\right)-p_{2}(\hat{z})}{n} \leq-\frac{1}{N_{1}} .
$$

Note que, $\Delta(z) \doteq p_{2} \circ \hat{f}(\hat{z})-p_{2}(\hat{z})$ para qualquer $\hat{z} \in \pi\left(p^{-1}(z)\right)$ é uma função (bem definida) contínua de $\mathbb{T}^{2}$. Seja $M>0$ um inteiro tal que $|\Delta(z)|<M$ para todo ponto $z$ do toro. Definindo

$$
\Delta^{n}(z)=\sum_{i=0}^{n-1} \Delta\left(f^{i}(z)\right),
$$

temos que $\left|\Delta^{n}(z)\right|<n M$. Desse modo, uma vez que

$$
\begin{gathered}
\Delta^{n}(z)=\Delta(z)+\Delta(f(z))+\ldots+\Delta\left(f^{n-1}(z)\right)=p_{2} \circ \hat{f}^{n}(\hat{z})-p_{2}(\hat{z}), \\
\left|\frac{p_{2}\left(f^{n}(\hat{z})\right)-p_{2}(\hat{z})}{n}\right|<M, \quad \forall n>0 .
\end{gathered}
$$


Assim, $\left\{\frac{p_{2}(\hat{f}(\hat{z}))-p_{2}(\hat{z})}{n}\right\}_{n \in \mathbb{N}^{*}} \subset[-M, M] \subset \mathbb{R}$ tem uma subsequência convergente. Portanto, se $\hat{z} \in B_{S}^{-}$, existe $\mathbb{N}_{1} \subset \mathbb{N}$ tal que

$$
\rho_{v}(\hat{f}) \ni \lim _{\mathbb{N}_{1} \ni n \rightarrow+\infty} \frac{p_{2}\left(\hat{f}^{n}(\hat{z})\right)-p_{2}(\hat{z})}{n} \leq-\frac{1}{N_{1}} .
$$

Dessa feita, existe $\epsilon>0$ tal que $\rho_{v}(\hat{f}) \supset[-\epsilon, 0]$.

Como foi dito antes, se $\omega\left(B_{S}^{-}\right)=\emptyset$ então $B_{S}^{-}$se comporta como se fosse o conjunto estável do ponto $S$, pois para todo ponto $\hat{z}$ de $B_{S}^{-}$,

$$
\lim _{n \rightarrow+\infty} p_{2}\left(\hat{f}^{n}(\hat{z})\right)-p_{2}(\hat{z})=-\infty .
$$

O conjunto $B_{S}$ foi definido como sendo o conjunto dos pontos que estão abaixo da horizontal pelo zero, $H$, e que permanecem em $H^{-}$por iteradas futuras de $\hat{f}$. Já $B_{S}^{-}$é a união de todas componentes conexas ilimitadas de $B_{S}$. Denotemos por $B_{S}^{-}($inv $)$ o análogo de $B_{S}^{-}$para $\hat{f}^{-1}$.

O próximo lema estabelece uma relação entre esses conjuntos.

Lema 3.2.3. Os conjuntos $\omega\left(B_{S}^{-}\right)$e $\omega\left(B_{S}^{-}(\right.$inv $\left.)\right)$são iguais.

Demonstração. Do lema 3.2.1 $\omega\left(B_{S}^{-}\right)$é invariante por $\hat{f}$. Assim, $\omega\left(B_{S}^{-}(i n v)\right)$ é invariante por $\hat{f}^{-1}$ (portanto, $f$-invariante). De fato, sendo

$$
\omega\left(B_{S}^{-}(i n v)\right)=\bigcap_{n=0}^{\infty}\left(\hat{f}^{-1}\right)^{n}\left(B_{S}^{-}(i n v)\right)=B_{S}^{-}(i n v) \cap \hat{f}^{-1}\left(B_{S}^{-}(i n v)\right) \cap \hat{f}^{-2}\left(B_{S}^{-}(i n v)\right) \cap \ldots
$$

e

$B_{S}^{-}(i n v)$ a união das componentes conexas ilimitadas de

$$
\bigcap_{n \leq 0}\left(\hat{f}^{-1}\right)^{n}\left(H^{-}\right)=H^{-} \cap \hat{f}\left(H^{-}\right) \cap \hat{f}^{2}\left(H^{-}\right) \cap \ldots,
$$

decorre que

$$
\hat{f}^{-1}\left(\omega\left(B_{S}^{-}(i n v)\right)\right)=\omega\left(B_{S}^{-}(i n v)\right),
$$

pois $\hat{f}^{-1}\left(B_{S}^{-}(i n v)\right) \subset B_{S}^{-}(i n v)$. Mostremos que esses conjuntos $\hat{f}$-invariantes são iguais.

Considere uma componente conexa de $\omega\left(B_{S}^{-}\right)$, digamos $\Gamma$. Como $\omega\left(B_{S}^{-}\right)$é um subconjunto de $H^{-}$e é (totalmente) invariante por $\hat{f}$, temos $\hat{f}^{-i}(\Gamma) \subset H^{-}$, para todo 
$i \in \mathbb{Z}$. Assim, $\Gamma \subset \bigcap_{i \geq 0} f^{i}\left(H^{-}\right)$e é um conexo ilimitado, $\log \mathrm{\Gamma} \subset B_{S}^{-}(i n v)$. Mas, (como feito para $\Gamma)$ para todo inteiro $n \geq 0, f^{n}(\Gamma)$ é uma componente conexa ilimitada de $\bigcap_{i \geq 0} \hat{f}^{\hat{i}}\left(H^{-}\right)$, então $\hat{f}^{n}(\Gamma) \subset B_{S}^{-}(i n v)$, para todo $n$ inteiro positivo. Desse modo,

$$
\Gamma \subset \bigcap_{n \leq 0} \hat{f}^{n}\left(B_{S}^{-}(i n v)\right)=\omega\left(B_{S}^{-}(i n v)\right) .
$$

Portanto, $\omega\left(B_{S}^{-}\right) \subset \omega\left(B_{S}^{-}(\right.$inv $\left.)\right)$. O mesmo raciocínio prova que $\omega\left(B_{S}^{-}\right) \supset \omega\left(B_{S}^{-}(\right.$inv $\left.)\right)$.

Tendo em mente esses resultados, podemos fornecer uma condição topológica para que um homeomorfismo Dehn twist tenha o zero como ponto interior do conjunto de rotação vertical de um levantamento fixado.

Teorema 3.2.1. Sejam $f \in D T\left(\mathbb{T}^{2}\right)$ e um levantamento $\hat{f} \in D T\left(S^{1} \times \mathbb{R}\right)$ tal que $0 \in \rho_{v}(\hat{f})$ e que o conjunto $\omega\left(B_{S}^{-}\right)$seja vazio. Então, zero é um ponto interior de $\rho_{v}(\hat{f})$.

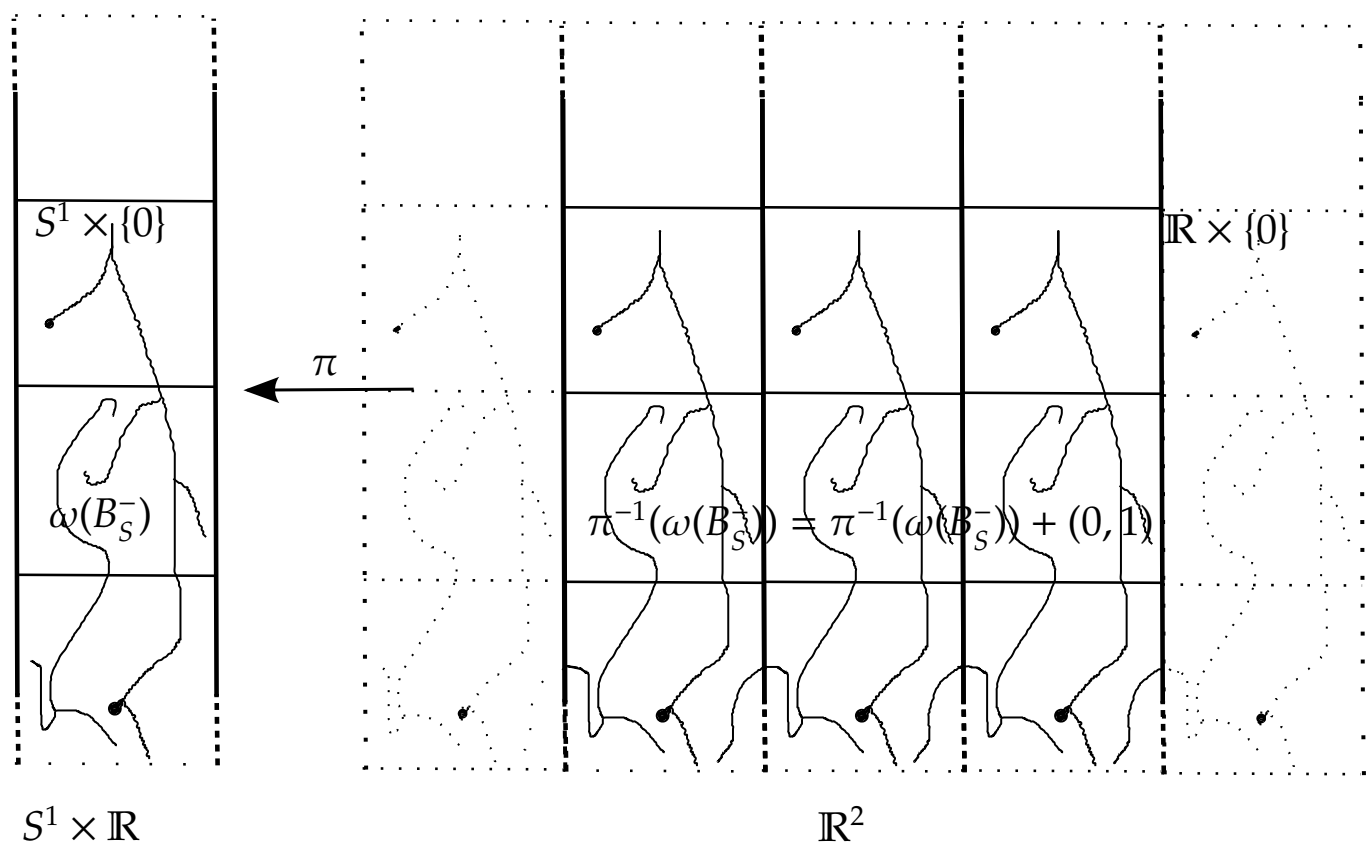

Figura 3.7: Levantamento de $\omega\left(B_{S}^{-}\right)$(vertical). 
Demonstração. Do teorema 3.1 .2 temos que $B_{S}^{-}$e $B_{S}^{-}($inv $)$são não vazios. Como $\omega\left(B_{S}^{-}\right)$ é vazio, o lema 3.2 .3 implica que, $\omega\left(B_{S}^{-}\right)(i n v)=\emptyset$. Nessas condições, aplicando o lema 3.2.2 duas vezes, obtemos que existe um $\epsilon>0$ tal que $\rho_{v}(\hat{f}) \supset[-\epsilon, \epsilon]$.

Resultados análogos valem para

$$
B_{N^{\prime}}^{+} B_{N}^{+}(i n v), \omega\left(B_{N}^{+}\right) \text {e } \omega\left(B_{N}^{+}(i n v)\right) .
$$

Os seguintes lemas esclarecem um pouco sobre a estrutura topológica desses conjuntos quando levantados para o plano pela aplicação de recobrimento do cilindro $\pi: \mathbb{R}^{2} \longrightarrow S^{1} \times \mathbb{R}$, veja a figura anterior.

Lema 3.2.4. Toda componente conexa $\tilde{\Gamma}$ de $\pi^{-1}\left(\omega\left(B_{S}^{-}\right)\right)$é ilimitada.

Demonstração. Seja Г̃ uma componente conexa de $\pi^{-1}\left(\omega\left(B_{S}^{-}\right)\right)$. Suponha, por contradição, que $\tilde{\Gamma} \subset$ interior $(Q)$, onde $Q$ é uma bola compacta. Veja a seguinte figura.
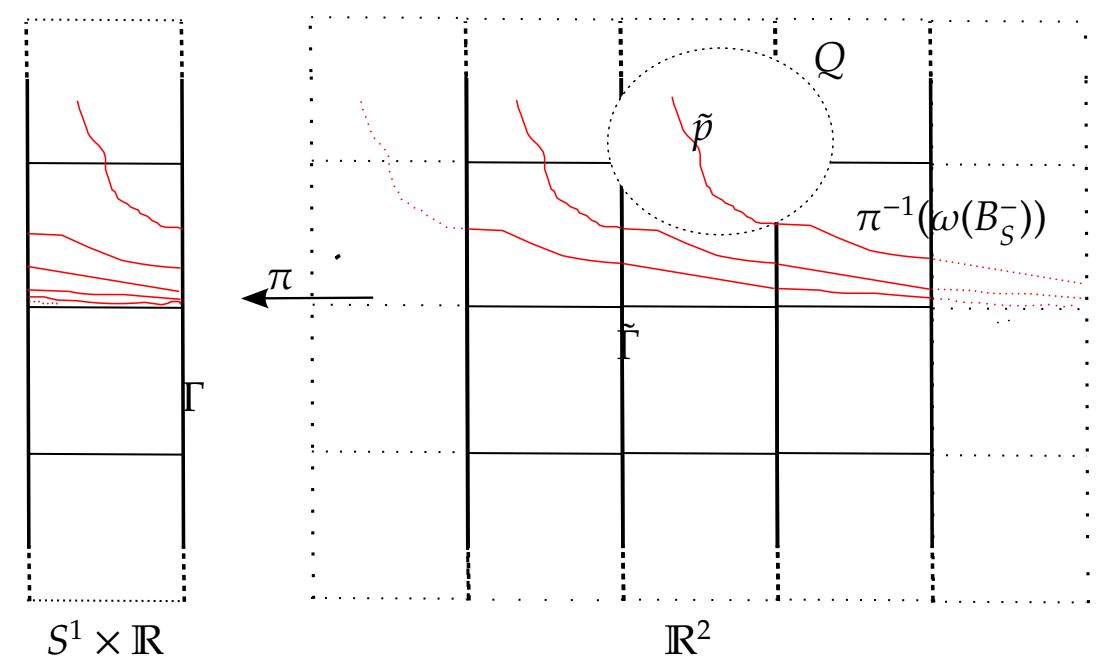

Figura 3.8: Levantamento de $\omega\left(B_{S}^{-}\right)$(horizontal).

Considere um ponto $\tilde{p} \in \tilde{\Gamma}$ e uma componente conexa de $\omega\left(B_{S}^{-}\right), \Gamma$, que contém $p=\pi(\tilde{p})$. É claro que por definição de $\omega\left(B_{S}^{-}\right)$, $\Gamma$ é ilimitado.

Denotemos por $V i z_{1 / n}(\Gamma)$ a $1 / n$-vizinhança de $\Gamma$, onde $n \in \mathbb{N}^{*}$. Como $V i z_{1 / n}(\Gamma)$ é aberto e conexo, seja $\gamma_{n}$ um arco simples de $\operatorname{Viz}_{1 / n}(\Gamma)$ começando em $p$ e terminando em um ponto de $H_{-n}$. 


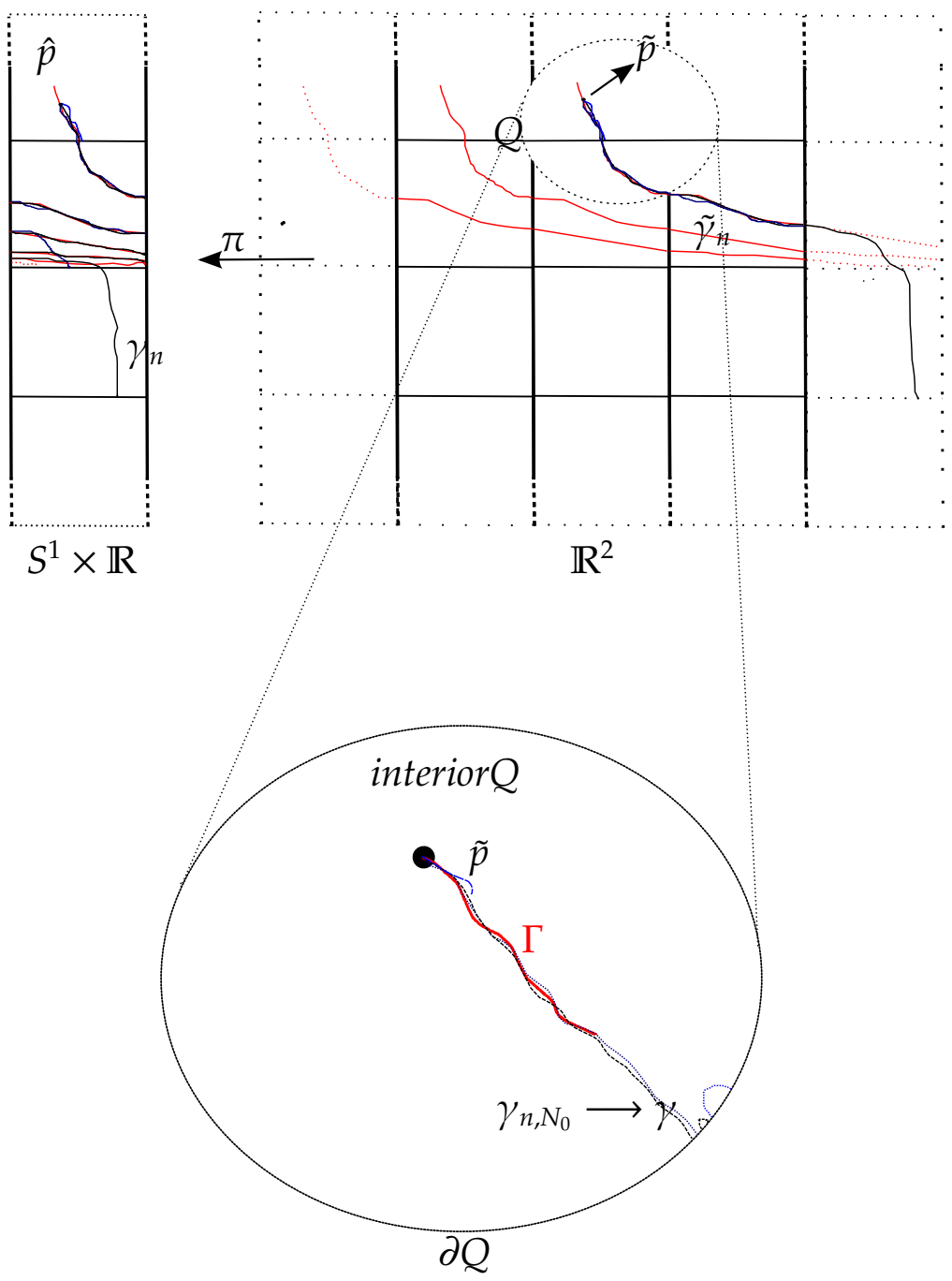

Figura 3.9: Levantamento dos arcos contínuos simples.

Seja $\tilde{\gamma}_{n}$ um levantamento de $\gamma_{n}$ que contém $\tilde{p}$. Como $Q$ é limitado, existe $N_{0}>1$ tal que, para todo inteiro $n \geq N_{0}$,

$$
\tilde{\gamma}_{n} \bigcap \partial Q \neq \emptyset
$$

Seja $\tilde{\gamma}_{n, N_{0}}$ a componente conexa de $\tilde{\gamma}_{n} \cap Q$ que contém $\tilde{p}$. Tomando uma subsequência convergente (na topologia de Hausdorff) de $\left\{\tilde{\gamma}_{n, N_{0}}\right\}_{n \in \mathbb{N}}$, temos que existe $\mathbb{N}_{1} \subset \mathbb{N}$ tal que $\left\{\tilde{\gamma}_{n, N_{0}}\right\}_{n \in \mathbb{N}_{1}}$ converge para um conjunto $\tilde{\gamma}$ compacto e conexo. Isto é, dado $\epsilon>0$ existe $N_{1}>0$ tal que para todo $\mathbb{N}_{1} \ni n \geq N_{1}$

$$
\tilde{\gamma}_{n, N_{0}} \subset V i z_{\epsilon}(\tilde{\gamma}) \text { e } \tilde{\gamma} \subset V i z_{\epsilon}\left(\tilde{\gamma}_{n, N_{0}}\right) \text {. }
$$


Se $\tilde{p} \notin \tilde{\gamma}$, então existe $\delta>0$ tal que $B_{\delta}(\tilde{p})$ (a bola de raio delta centrada em $\tilde{p}$ ) não intercepta $V_{\delta}(\tilde{\gamma})$. Mas para $n \in \mathbb{N}_{1}$ suficientemente grande, $\gamma_{n, N_{0}}$ está contido em $V_{\delta}(\tilde{\gamma})$. O que é um absurdo, pois $\gamma_{n, N_{0}}$ contém $\tilde{p}$. Portanto, $\tilde{p} \in \tilde{\gamma}$. Analogamente, pode-se mostrar que $\tilde{\gamma} \cap \partial Q \neq \emptyset$. Assim, $\tilde{p} \in \tilde{\gamma}$ e $\partial Q \cap \tilde{\gamma} \neq \emptyset$.

Sejam $\hat{z} \in \pi(\tilde{\gamma})$ e $\epsilon>0$. Assim, existe $\left\{\hat{z}_{n} \subset \gamma_{n}\right\}$ tal que $d\left(\hat{z}_{n}, \hat{z}\right)<\epsilon$, para $n>N_{1}$. Por construção $\gamma_{n} \subset V i z_{\frac{1}{n}}(\Gamma)$, logo existe $\hat{w} \in \Gamma$ tal que $d\left(\hat{w}, \hat{z}_{n}\right)<\frac{1}{n}$. Nessas condições, pela desigualdade triangular e pela arbitrariedade de $\epsilon, \hat{z} \in \Gamma$, pois $d(\hat{z}, \hat{w})<\epsilon+1 / n$, para todo inteiro $n$ suficientemente grande. Portanto, $\pi(\tilde{\gamma}) \subset \Gamma$. Assim, $\tilde{\gamma} \subset \pi^{-1}(\Gamma) \subset$ $\pi^{-1}\left(\omega\left(B_{S}^{-}\right)\right)$. Como $\tilde{\gamma} \cap \tilde{\Gamma} \neq \emptyset$, isso contradiz o fato de termos tomado $\tilde{\Gamma}$ como sendo uma componente conexa de $\pi^{-1}\left(\omega\left(B_{S}^{-}\right)\right)$. Dessa feita, $\tilde{\Gamma}$ escapa de qualquer compacto, donde segue que é ilimitada.

Lema 3.2.5. Seja $\tilde{\Gamma}$ uma componente conexa de $\pi^{-1}\left(\omega\left(B_{S}^{-}\right)\right)$, então $\tilde{\Gamma}^{c}$ é conexo.

Demonstração. Observe que $\mathbb{R}^{2} \backslash \tilde{\Gamma}$ tem pelo menos uma componente conexa que contém $\mathbb{R} \times[0,+\infty[$.

Se existir outra componente, $O$, ela deve ser um subconjunto de $\mathbb{R} \times]-\infty, 0]$. Como $\pi^{-1}\left(\omega\left(B_{S}^{-}\right)\right)$é um conjunto fechado e $\tilde{f}$ invariante,

$$
\left.\left.\tilde{f}^{n}(O) \subset \mathbb{R} \times\right]-\infty, 0\right],
$$

para todo inteiro $n$. Sendo $\tilde{\Gamma} \cup O$ um conjunto conexo, fechado e ilimitado,

$$
\tilde{\Gamma} \bigcup O \subset \pi^{-1}\left(B_{S}^{-}\right) .
$$

Ainda,

$$
\tilde{\Gamma} \bigcup O \subset \pi^{-1}\left(\omega\left(B_{S}^{-}\right)\right),
$$

pois $\tilde{f}^{-i}(\tilde{\Gamma} \cup O)=\tilde{f}^{-i}(\tilde{\Gamma}) \cup \tilde{f}^{-i}(O)=\tilde{\Gamma}_{i} \cup O_{i}$, onde $\tilde{\Gamma}_{i}$ é uma componente conexa de $\pi^{-1}\left(\omega\left(B_{S}^{-}\right)\right)$e $\left.\left.O_{i} \subset \mathbb{R} \times\right]-\infty, 0\right]$ conexo tal que $\partial O_{i} \subset \tilde{\Gamma}_{i}$. De novo, pela invariância de $\pi^{-1}\left(\omega\left(B_{S}^{-}\right)\right), \tilde{\Gamma}_{i} \cup O_{i}$ é um subconjunto de $\pi^{-1}\left(B_{S}^{-}\right)$. Em outras palavras,

$$
\tilde{\Gamma} \bigcup O \subset \tilde{f}^{\tilde{1}}\left(\pi^{-1}\left(B_{S}^{-}\right)\right),
$$

para todo inteiro positivo $i$. Nessas condições, $\tilde{\Gamma} \cup O \subset \bigcap_{i \geq 0} \tilde{f}^{i}\left(\pi^{-1}\left(B_{S}^{-}\right)\right)=\pi^{-1}\left(\omega\left(B_{S}^{-}\right)\right)$.

Lema 3.2.6. O complementar de $\pi^{-1}\left(\omega\left(B_{S}^{-}\right)\right)$é conexo.

Demonstração. Análogo a demonstração anterior. 
Em suma, as principais propriedades de $\omega\left(B_{S}^{-}\right)$são:

- $\hat{f}\left(\omega\left(B_{S}^{-}\right)\right)=\omega\left(B_{S}^{-}\right)$;

- $\mathbb{R}^{2} \backslash \pi^{-1}\left(\omega\left(B_{S}^{-}\right)\right)$é conexo;

- Para todo $n_{1} \in \mathbb{Z}, \pi^{-1}\left(\omega\left(B_{S}^{-}\right)\right)=\pi^{-1}\left(\omega\left(B_{S}^{-}\right)\right)+\left(n_{1}, 0\right)$;

- Para todo $n_{2} \in \mathbb{N}, \pi^{-1}\left(\omega\left(B_{S}^{-}\right)\right)-\left(0, n_{2}\right) \subset \pi^{-1}\left(\omega\left(B_{S}^{-}\right)\right)$;

- Para todo $n_{2} \in \mathbb{Z}, \pi^{-1}\left(\omega\left(B_{S}^{-}\right)\right)+\left(0, n_{2}\right)$ é $\tilde{f}$-invariante;

Claramente, todos os resultados obtidos até aqui possuem uma versão similar quando considerado o conjunto $B_{N}^{+}$.

\subsection{Homeomorfismos de Brouwer}

Nessa seção apresentaremos alguns resultados da teoria de Brouwer para homeomorfismos do plano. A teoria de Brouwer trata de homeomorfismos de $\mathbb{R}^{2}$ (que preservam orientação) sem pontos fixos, e tem como objetivo, entre outras coisas, a caracterização desses em termos de conjugação topológica com uma translação do plano. Veja [19], [15], [20] e [17].

Definição 3.3.1. Uma aplicação $\tilde{h}: \mathbb{R}^{2} \longrightarrow \mathbb{R}^{2}$ é dita ser uma aplicação de Brouwer se $\tilde{h}$ for um homeomorfismo que preserva a orientação sem pontos fixos.

Seguem alguns conceitos da teoria de Brouwer:

1. $\alpha \subset \mathbb{R}^{2}$ é um arco de translação, se $\alpha$ é um arco simples tal que seus pontos finais são $\tilde{z} \in \mathbb{R}^{2}$ e $\tilde{h}(\tilde{z})$ e ainda $\alpha \cap \tilde{h}(\alpha)=\tilde{h}(\tilde{z})$ ou $\alpha \cap \tilde{h}(\alpha)=\{\tilde{z}, \tilde{h}(\tilde{z})\}$.

2. Um subconjunto $E \subset \mathbb{R}^{2}$ é denominado livre se $E \cap \tilde{h}(E)=\emptyset$.

3. Uma linha de Brouwer $L \subset \mathbb{R}^{2}$ é a imagem de uma imersão própria fechada $l: \mathbb{R} \longrightarrow \mathbb{R}^{2}$, tal que: $L=l(\mathbb{R})$ divide o plano em duas componentes conexas e $\tilde{h}(L)$ pertence a uma dessas componentes, enquanto que $\tilde{h}^{-1}(L)$ pertence a outra.

Lema 3.3.1. (Lema de Brouwer para arcos de translação) Seja $\tilde{h}: \mathbb{R}^{2} \longrightarrow \mathbb{R}^{2}$ uma aplicação de Brouwer. Seja $\tilde{z} \in \mathbb{R}^{2}$, então existe um arco de translação, $\alpha$, para $\tilde{h}$ tal que $\tilde{z} \in$ interior $(\alpha)$ e $\alpha \cap \tilde{h}^{n}(\alpha)=\emptyset$, para todo $n \notin\{-1,0,1\}$. 
Segue um importante corolário.

Corolário 3.3.2. Seja $\tilde{h}: \mathbb{R}^{2} \longrightarrow \mathbb{R}^{2}$ uma aplicação de Brouwer. Dado um conjunto $E \subset \mathbb{R}^{2}$ conexo por caminhos e livre, então $E \cap \tilde{h}^{n}(E)=\emptyset$, para todo $n \in \mathbb{Z}^{*}$.

Teorema 3.3.3. (Teorema de translação do plano de Brouwer) Seja $\tilde{h}: \mathbb{R}^{2} \longrightarrow \mathbb{R}^{2}$ uma aplicação de Brouwer. Para todo ponto $\tilde{z} \in \mathbb{R}^{2}$, existe uma linha de Brouwer $L$ passando por $\tilde{z}$.

\subsection{Decomposição do plano por tijolos}

Nessa seção apresentaremos a definição de decomposição do plano por tijolos, que será fundamental na demonstração do teorema 1.3.2.

Definamos a decomposição do plano por tijolos como sendo:

$$
\mathbb{R}^{2}=\bigcup_{i \geq 0} D_{i}
$$

onde cada $D_{i} \in$ Decomposição por Tijolos é o fecho de um conjunto aberto, conexo e simplesmente conexo tal que $\partial D_{i}$ é uma curva simples poligonal e

$$
\operatorname{interior}\left(D_{i}\right) \cap \text { interior }\left(D_{j}\right)=\emptyset \text {, }
$$

para $i \neq j$. A decomposição é dita localmente finita quando $\bigcup_{i \geq 0} \partial D_{i}$ é um grafo cujos vertices possuem três lados adjacentes e o número de elementos da decomposição contida em qualquer compacto do plano é finito. Diremos que a decomposição é livre se cada tijolo é livre, ou seja, $\tilde{h}\left(D_{i}\right) \cap D_{i}=\emptyset$, para todo $i \in \mathbb{N}$.

Dados dois tijolos, $D$ e $E$, diremos que existe uma cadeia ligando $D$ a $E$ se existirem tijolos

$$
D=B_{0}, B_{1}, B_{2}, \ldots, B_{n-1}, B_{n}=E,
$$

tais que $\tilde{h}\left(B_{i}\right) \cap B_{i+1} \neq \emptyset$, para $i=0,1,2, \ldots, n-1$. Uma cadeia é dita livre se $B_{i}$ é livre para todo $i=0,1, \ldots, n$ e é dita fechada (ou periódica) se $D=E$. O seguinte lema é uma versão do teorema de Franks (ver [10]) devido a Le Roux [15].

Lema 3.4.1. Se $\tilde{h}$ é um homeomorfismo do plano que preserva orientação, a existência de uma cadeia fechada de tijolos livres implica que existe uma curva fechada simples $\gamma \subset \mathbb{R}^{2}$, tal que

$$
\operatorname{indice}(\gamma, \tilde{h})=\operatorname{grau}\left(\gamma, \frac{\tilde{h}(\tilde{z})-\tilde{z}}{\|\tilde{h}(\tilde{z})-\tilde{z}\|}\right)=1 \text {. }
$$


O indice $(\gamma, \tilde{h})$ é o número de voltas do campo vetorial $\tilde{h}(\tilde{z})-\tilde{z}$ sobre $\gamma$, onde $\gamma: S^{1} \longrightarrow \mathbb{R}^{2}$ ou, alternativamente, o grau da aplicação $H: S^{1} \longrightarrow S^{1}$ dada por $H(t)=\tilde{h}(\gamma(t))-\gamma(t) /\|\tilde{h}(\gamma(t))-\gamma(t)\|$. Note que indice $(\gamma, \tilde{h}) \neq 0$ implica a existência de um ponto fixo, para $\tilde{h}$, na componente limitada do complementar de $\gamma\left(S^{1}\right)$. 
Nessa seção apresentaremos as demonstrações dos resultados de 1.3 . Antes de mais nada, lembremos que um conjunto $K \subset S^{1} \times \mathbb{R}$ é um continuum essencial se $K$ for compacto, conexo e $S^{1} \times \mathbb{R} \backslash K$ tiver duas componentes conexas ilimitadas ${ }^{1}$.

\subsection{Demonstração do teorema 1.3.2}

Demonstração. Sejam $f$ um homeomorfismo homotópico a um Dehn twist e $\hat{f}$ um levantamento de $f$ para o cilindro (vertical). Quer-se mostrar que

$$
\rho_{v}(\hat{f})=\{0\} \text { se, e somente se, existe } K \text {, um continuum essencial } \hat{f} \text {-invariante. }
$$

Suponha que exista $K$ tal como acima. Sendo $K$ compacto, existe um inteiro $M_{0}>0$ tal que $K \subset S^{1} \times\left[-M_{0}, M_{0}\right]$. Sejam $K^{-}$e $K^{+}$as componentes conexas de $\left(S^{1} \times \mathbb{R}\right) \backslash K$ que contém, respectivamente, o fim inferior e o fim superior de $S^{1} \times \mathbb{R}$.

Como $K$ é invariante por $\hat{f}$ temos que $K^{-}$e $K^{+}$são $\hat{f}$-invariantes. Nessas condições, existe um inteiro $M \geq M_{0}$ tal que

$$
K^{-} \bigcap\left(K^{+}-(0, M)\right)
$$

é um conjunto aberto, conexo, $\hat{f}$-invariante que contém um domínio fundamental. Portanto, para todo ponto $\hat{z} \in S^{1} \times \mathbb{R}$ e para todo inteiro $n>0$

$$
\left|p_{2} \circ \hat{f}^{n}(\hat{z})-p_{2}(\hat{z})\right|<M .
$$

${ }^{1}$ veja a figura 1.3 
Por definição do conjunto de rotação vertical de $\hat{f}$, temos que $v \in \rho_{v}(\hat{f})$ se, e somente se, existem subsequências $\left\{n_{i}\right\} \subset \mathbb{N}$ e $\left\{\hat{z}_{i}\right\} \subset S^{1} \times \mathbb{R}$ tais que

$$
v=\lim _{i \rightarrow \infty} \frac{p_{2} \circ \hat{f}^{n_{i}}\left(\hat{z}_{i}\right)-p_{2}\left(\hat{z}_{i}\right)}{n_{i}},
$$

portanto, $v=0$. Logo, $\rho_{v}(\hat{f})=\{0\}$.

Suponha agora que $\rho_{v}(\hat{f})=\{0\}$. Observe que, se

$$
\rho_{v}(\hat{f})=\{0\} \text { implicar que } \bigcup_{n \geq 0} \hat{f}^{n}(H) \text { é limitado, }
$$

então concluímos a tese, isto é, existe $K$ continuum essencial invariante por $\hat{f}$. De fato, suponhamos que

$$
\bigcup_{n \geq 0} \hat{f}^{n}(H) \text { seja limitado. }
$$

Assim, existe $M>0$ tal que para todo ponto $\hat{z} \in S^{1} \times \mathbb{R}$ e para todo inteiro $n>0$

$$
\left|p_{2} \circ \hat{f}^{n}(\hat{z})-p_{2}(\hat{z})\right|<M .
$$

Se existir $\hat{z}_{0} \in S^{1} \times \mathbb{R}$ tal que

$$
\left|p_{2} \circ \hat{f}^{-n_{0}}\left(\hat{z}_{0}\right)-p_{2}\left(\hat{z}_{0}\right)\right|>M, \text { para algum inteiro } n_{0}>0,
$$

então, definindo $\hat{w}_{0}=\hat{f}^{-n_{0}}\left(\hat{z}_{0}\right)$, temos que

$$
\left|p_{2} \circ \hat{f}^{n_{0}}\left(\hat{w}_{0}\right)-p_{2}\left(\hat{w}_{0}\right)\right|>M, \text { para algum inteiro } n_{0}>0,
$$

o que é um absurdo. Nessas condições, a órbita de qualquer ponto do cilindro é uniformemente limitada, ou seja,

$$
\bigcup_{n \in \mathbb{Z}} \hat{f}^{n}\left(S^{1} \times[0,1]\right) \text { é limitado. }
$$

Considere o conjunto

$$
O=\bigcup_{n \in \mathbb{Z}} \hat{f}^{n}\left(S^{1} \times\right]-\infty, 0[)
$$

Temos que $O$ é um subconjunto de $\left.\left.S^{1} \times\right]-\infty, M\right]$ aberto, conexo e $\hat{f}$-invariante. Ainda, existe uma única componente do bordo de $O$ contida em $S^{1} \times[0, M]$ que é compacta, 
conexa e que separa os fins do cilindro. Denotemos essa componente por $K^{*}$. Como $\hat{f}(O)=O$ temos, $\hat{f}\left(K^{*}\right)=K^{*}$.

Portanto, para finalizar o teorema basta mostrar que, sob as suas hipóteses, $\bigcup_{n \geq 0} \hat{f}^{n}(H)$ é sempre limitado.

Sendo assim, suponhamos, por contradição, que $\bigcup_{n \geq 0} \hat{f}^{n}(H)$ seja ilimitado.

Desse modo, podemos supor, sem perda de generalidade, que para cada $M>0$, existem um ponto $\hat{z} \in S^{1} \times \mathbb{R}$ e um inteiro positivo $n$ tal que

$$
p_{2} \circ \hat{f}^{n}(\hat{z})-p_{2}(\hat{z})>M .
$$

Como $\rho_{v}(\hat{f})=\{0\}$, temos que, pelo teorema 3.1.2. $B_{N}^{+}$e $B_{S}^{-}$são não vazios. Além disso, pelo lema 3.2.2.

$$
\omega\left(B_{N}^{+}\right) \neq \emptyset \quad \text { e } \quad \omega\left(B_{S}^{-}\right) \neq \emptyset,
$$

pois, caso contrário o conjunto de rotação vertical de $\hat{f}$ teria interior não vazio.

Seja $\tilde{f}$ um levantamento de $\hat{f}$ para $\mathbb{R}^{2}$. Seguem algumas observações sobre $\tilde{f}$.

Observação 4.1.1. Sendo $\hat{f} \in D T\left(\mathbb{T}^{2}\right), \tilde{f} \in D T\left(\mathbb{R}^{2}\right)$ pode ser escrito na forma, se $(\tilde{x}, \tilde{y}) \in \mathbb{R}^{2}$,

$$
\tilde{f}(\tilde{x}, \tilde{y})=(\tilde{x}+m \tilde{y}, \tilde{y})+\Phi(\tilde{x}, \tilde{y}),
$$

onde $m \in \mathbb{N}^{*}$ e $\Phi(\tilde{x}+1, \tilde{y}+1)=\Phi(\tilde{x}, \tilde{y})$, para todo $(\tilde{x}, \tilde{y}) \in \mathbb{R}^{2}$. Portanto, existe um número real $M_{\text {Dehn }}>0$ tal que

$$
p_{1} \circ \tilde{f}(\tilde{x}, \tilde{y})>\tilde{x}+1 \text { se } \tilde{y}>M_{D e h n}
$$

$\mathrm{e}$

$$
p_{1} \circ \tilde{f}(\tilde{x}, \tilde{y})<\tilde{x}-1 \text { se } \tilde{y}<-M_{\text {Dehn }} .
$$

Observação 4.1.2. Outra propriedade decorrente de $f$ ser um Dehn twist é que existem dois números $V_{f}>0$ e $A_{f}>0$ tais que para todo conjunto compacto conexo $G \subset \mathbb{R}^{2}$ satisfazendo

$$
\left|p_{2}(G)\right|:=\left.\max p_{2}\right|_{G}-\left.\min p_{2}\right|_{G}>V_{f} \quad \text { (Comprimento Vertical Máximo de } G>V_{f} \text { ) }
$$

e

$\left|p_{1}(G)\right|:=\left.\max p_{1}\right|_{G}-\left.\min p_{1}\right|_{G}<1, \quad($ Comprimento Horizontal Máximo de $G<1$ ) então 


$$
\left|p_{1}(\tilde{f}(G))\right|>2 \text { e }\left.\quad p_{2}\right|_{\tilde{f}(G)}>\left.\min p_{2}\right|_{G}-A_{f} .
$$

Essas observações são consequências de $\Phi: \mathbb{R}^{2} \longrightarrow \mathbb{R}^{2}$ ser limitado em todo plano e de $\tilde{f}: \mathbb{R}^{2} \longrightarrow \mathbb{R}^{2}$ ter uma condição de twist infinito, isto é,

$$
\lim _{\tilde{y} \longrightarrow \pm \infty} p_{2} \circ \tilde{f}(\tilde{x}, \tilde{y}) \longrightarrow \pm \infty .
$$

O próximo lema é um resultado técnico que permite provar que existe um transladado vertical inteiro de $\omega\left(B_{S}^{-}\right)$que intercepta toda semi-reta vertical ilimitada superiormente. Em outras palavras, dado $M>0$ existe $N_{1}>0$ tal que, para todo $\hat{x} \in S^{1}$,

$$
\left(\omega\left(B_{S}^{-}\right)+\left(0, N_{1}\right)\right) \bigcap\{\hat{x}\} \times[M,+\infty[\neq \emptyset .
$$

Considere as funções $\mu: S^{1} \supset \operatorname{dom}(\mu) \longrightarrow \mathbb{R}$ e $v: S^{1} \supset \operatorname{dom}(v) \longrightarrow \mathbb{R}$ definidas por:

$$
\mu(\hat{x})=\max \left\{\hat{y} \in \mathbb{R}:(\hat{x}, \hat{y}) \in \omega\left(B_{S}^{-}\right)\right\}
$$

e

$$
v(\hat{x})=\min \left\{\hat{y} \in \mathbb{R}:(\hat{x}, \hat{y}) \in \omega\left(B_{N}^{+}\right)\right\} .
$$

Lema 4.1.1. As funções $\mu$ e $v$ possuem domínios iguais a $S^{1}$, isto é,

$$
\operatorname{dom}(\mu)=\operatorname{dom}(v)=S^{1} .
$$

E, existe uma constante $M_{f}>0$ tal que

$$
\max _{\hat{x}, \hat{y} \in S^{1}}|\mu(\hat{x})-\mu(\hat{y})|<M_{f}
$$

e

$$
\max _{\hat{x}, \hat{y} \in S^{1}}|v(\hat{x})-v(\hat{y})|<M_{f} .
$$

Demonstração. A prova é análoga para ambos os casos, então consideremos somente a função $\left.\left.\mu: S^{1} \supset \operatorname{dom}(\mu) \longrightarrow\right]-\infty, 0\right] \subset \mathbb{R}$.

Suponha que exista $\hat{x}_{0} \in S^{1}$ tal que $\mu$ não esteja definido. Dessa forma, existe $\tilde{x}_{0} \in \mathbb{R}$ tal que

$$
\pi^{-1}\left(\omega\left(B_{S}^{-}\right)\right) \bigcap\left\{\tilde{x}_{0}\right\} \times \mathbb{R}=\emptyset .
$$


Seja $\Gamma$ uma componente conexa de $\pi^{-1}\left(\omega\left(B_{S}^{-}\right)\right)$contida em $\left.] \tilde{x}_{0}, \tilde{x}_{0}+1[\times]-\infty, 0\right]$. Assim, a partir do lema 3.2.4. $\Gamma$ é ilimitado verticalmente. Seja $\left.\tilde{x}_{1} \in\right] \tilde{x}_{0}, \tilde{x}_{0}+1$ [ tal que $\mu(\hat{x}) \leq \mu\left(\hat{x}_{1}\right)$, para todo $\hat{x}=\tilde{x} \bmod 1 \in \operatorname{dom}(\mu)$, onde $\hat{x}_{1}=\tilde{x}_{1} \bmod 1$. Considere $\Gamma_{V_{f}}$ como sendo a componente conexa de

$$
\Gamma \bigcap\left[\tilde{x}_{0}, \tilde{x}_{0}+1\right] \times\left[\mu\left(\hat{x}_{1}\right)-V_{f}, \mu\left(\hat{x}_{1}\right)\right],
$$

que contém o ponto $\left(\tilde{x}_{1}, \mu\left(\hat{x}_{1}\right)\right)$.

Desse modo,

$$
\left|p_{1}\left(\Gamma_{V_{f}}\right)\right|<1 \text { e }\left|p_{2}\left(\Gamma_{V_{f}}\right)\right|>V_{f},
$$

assim, pela observação 4.1 .2 acima,

$$
\left|p_{1}\left(\tilde{f}\left(\Gamma_{V_{f}}\right)\right)\right|>2,
$$

o que contradiz 4.2. Logo, $\mu$ está bem definida em $S^{1}$.

$\mathrm{O}$ feito acima também prova a existência da constante $M_{f}>0$ do lema. De fato, como $\omega\left(B_{S}^{-}\right)$é fechado, para cada $\hat{x} \in S^{1}$ temos $(\hat{x}, \mu(\hat{x})) \in \omega\left(B_{S}^{-}\right)$. Seja $\tilde{x}_{0} \in \mathbb{R}$ tal que $\mu(\hat{x}) \leq \mu\left(\hat{x}_{0}\right)$, para todo $\hat{x}=\tilde{x} \bmod 1 \in S^{1}$, onde $\hat{x}_{0}=\tilde{x}_{0} \bmod 1$. Seja $\Gamma_{0}$ a componente conexa de $\omega\left(B_{S}^{-}\right)$que contém o ponto $\left(\hat{x}_{0}, \mu\left(\hat{x}_{0}\right)\right)$.

Se existir $\tilde{\Gamma}_{V_{f}}$ componente conexa de

$$
\pi^{-1}\left(\Gamma_{0}\right) \bigcap \mathbb{R} \times\left[\mu\left(\hat{x}_{0}\right)-V_{f}, \mu\left(\hat{x}_{0}\right)\right]
$$

tal que $\left|p_{1}\left(\tilde{\Gamma}_{V_{f}}\right)\right|>1$, temos que para todo $\hat{x} \in S^{1}$,

$$
\mu\left(\hat{x}_{0}\right)-V_{f} \leq \mu(\hat{x}) \leq \mu\left(\hat{x}_{0}\right) .
$$

Portanto, para todo $\hat{x} \in S^{1}, 0 \leq \mu\left(\hat{x}_{0}\right)-\mu(\hat{x}) \leq V_{f}$. E, nesse caso, tomamos $M_{f}=V_{f}$.

Caso contrário, teríamos que $\left|p_{1}\left(\tilde{\Gamma}_{V_{f}}\right)\right| \leq 1$. Mas pela observação 4.1.2.

$$
\left|p_{1}\left(\tilde{f}\left(\tilde{\Gamma}_{V_{f}}\right)\right)\right|>2 \text { e }\left.p_{2}\right|_{\tilde{f}\left(\tilde{\Gamma}_{V_{f}}\right)} \geq\left.\min p_{2}\right|_{\tilde{\Gamma}_{V_{f}}}-A_{f}
$$

Sendo $\pi^{-1}\left(\omega\left(B_{S}^{-}\right)\right) \tilde{f}$-invariante, para todo $\hat{x} \in S^{1}$

$$
\mu(\hat{x}) \geq\left.\min p_{2}\right|_{\tilde{\Gamma}_{V_{f}}}-A_{f}=\mu\left(\hat{x}_{0}\right)-V_{f}-A_{f} .
$$

Logo, $0 \leq \mu\left(\hat{x}_{0}\right)-\mu(\hat{x}) \leq V_{f}+A_{f}$. Desse modo,

$$
\max _{\hat{x}, \hat{y} \in S^{1}}|\mu(\hat{x})-\mu(\hat{y})|<M_{f}
$$

onde $M_{f} \geq V_{f}+A_{f}$. 
A construção a seguir será análoga para $\omega\left(B_{S}^{-}\right)$e $\omega\left(B_{N}^{+}\right)$, sendo assim apresentaremos os detalhes apenas para $\omega\left(B_{S}^{-}\right)$. Iremos considerar uma translação vertical (inteira) de $\omega\left(B_{S}^{-}\right)$tal que intercepte toda semi-reta vertical, iniciando em $M_{\text {Dehn }}$ (figurado na observação 4.1.1), ilimitada superiormente. Inicialmente, note que para todo $\hat{x} \in S^{1}$,

$$
\mu(\hat{x})+\left\lfloor-\sup _{\hat{z} \in S^{1}} \mu(\hat{z})+M_{f}+M_{\text {Dehn }}+1\right\rfloor>M_{\text {Dehn }} .
$$

Veja figura 4.1 abaixo.

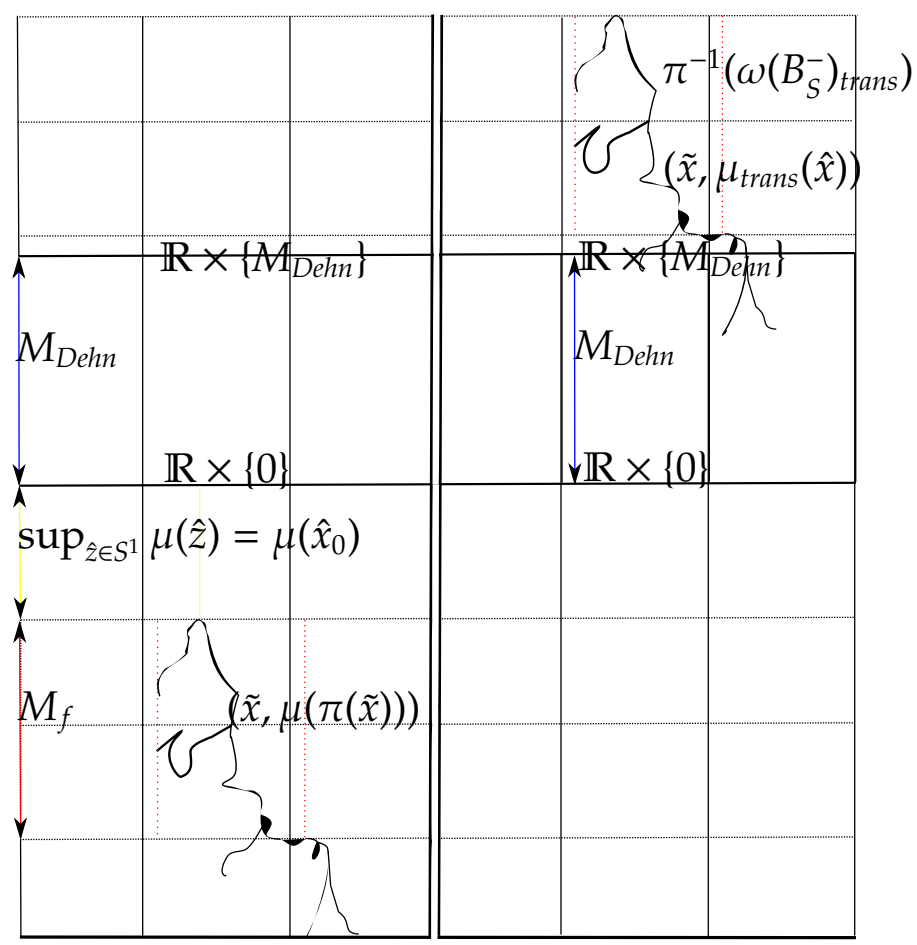

Figura 4.1: Transladado vertical de $\pi^{-1}\left(\omega\left(B_{S}^{-}\right)\right)$.

Assim,

$$
\left.\omega\left(B_{S}^{-}\right)_{\text {trans }}:=\omega\left(B_{S}^{-}\right)+\left(0, L-\sup _{z \in S^{1}} \mu(\hat{z})+M_{f}+M_{\text {Dehn }}+1\right\rfloor\right)
$$

é um transladado vertical de $\omega\left(B_{S}^{-}\right)$pelo inteiro positivo $\left\lfloor-\sup _{\hat{\varepsilon} \in S^{1}} \mu(\hat{z})+M_{f}+M_{\text {Dehn }}+1\right\rfloor$ tal que, para todo $\hat{x} \in S^{1}$, existe $(\hat{x}, \hat{y}) \in \omega\left(B_{S}^{-}\right)_{\text {trans }} \operatorname{com} \hat{y}>M_{\text {Dehn }}$. Em outras palavras, a função $\mu$ associada a $\omega\left(B_{S}^{-}\right)_{\text {trans }}$, denotada por $\mu_{\text {trans }}$, satisfaz

$$
\mu_{\text {trans }}(\hat{x})>M_{\text {Dehn }} \text {, para todo } \hat{x} \in S^{1} .
$$


Considere $\tilde{x} \in \mathbb{R}$ e a semi-reta vertical $\{\tilde{x}\} \times] \mu_{\text {trans }}(\hat{x}),+\infty[$. Então,

$$
\pi^{-1}\left(\omega\left(B_{S}^{-}\right)_{\text {trans }}\right) \bigcap v=\emptyset .
$$

Seja $\Theta$ a componente conexa de $\pi^{-1}\left(\omega\left(B_{S}^{-}\right)_{\text {trans }}\right)$ que contém o ponto $\left(\tilde{x}, \mu_{\text {trans }}(\hat{x})\right)$. $\Theta \cup v$ possui algumas propriedades topológicas e dinâmicas de importância fundamental na conclusão da tese. Isso é o conteúdo do seguinte lema.

Lema 4.1.2. $\Theta \cup v$ é um conjunto fechado, conexo, $\mathbb{R}^{2} \backslash(\Theta \cup v)$ tem, exatamente, duas componentes conexas, sendo uma delas positivamente invariante e $\tilde{f}^{n}(v) \cap v=\emptyset$, para todo inteiro $n \neq 0$.

Demonstração. Por construção, $\Theta \cap v=\emptyset \mathrm{e} \Theta \cap \bar{v}$ é um único ponto de $\Theta$, assim como $\Theta$ e $v$ são conexos, $\Theta \cup v$ é conexo. Ainda, o fecho $\overline{\Theta \cup v}=\bar{\Theta} \cup \bar{v}=\Theta \cup \bar{v}=\Theta \cup v$, logo fechado.

Mostremos que $(\Theta \cup v)^{c}$ tem exatamente duas componentes conexas a quais denotaremos por $O_{D}$ e $O_{E}$. Para cada ponto $\tilde{z} \in v$, existe um $\delta>0$ tal que $B_{\delta}(\tilde{z}) \cap \Theta=\emptyset$, onde $B_{\delta}(\tilde{z})$ é a bola aberta de raio $\delta$ e centro $\tilde{z}$. Entretanto, $B_{\delta}(\tilde{z}) \backslash v$ tem exatamente duas componentes conexas, uma do lado esquerdo de $v$, contida em $O_{E}$, e a outra do lado direito de $v$, contida em $O_{D}$.

Suponha, por contradição, que exista $O$ uma terceira componente conexa do complementar de $(\Theta \cup v)^{c}$. Como os pontos que estão suficientemente próximos de $v$, mas não pertencendo a $v$, estão em $O_{E} \cup O_{D}$, temos que $\partial O$ não intercepta $v$. Portanto, $\partial O \subset \Theta$. Sendo $\Theta$ componente conexa de $\omega\left(B_{S}^{-}\right)_{\text {trans }}$,

$$
\left.\Theta \subset \mathbb{R} \times]-\infty,\left[-\sup _{\hat{z} \in S^{1}} \mu(\hat{z})+M_{f}+M_{\text {Dehn }}+1\right]\right] .
$$

Mas aplicando o lema 3.2.5. $\Theta^{c}$ é conexo, donde segue uma contradição que implica que

$$
(\Theta \cup v)^{c}=O_{E} \cup O_{D} .
$$

Supondo como anteriormente, que o número $m \in \mathbb{Z}^{*}$, que define a classe de isotopia Dehn twist, seja positivo, podemos provar que $O_{D}$ é positivamente invariante. Com efeito, por construção de $v$

$$
\tilde{f}(v) \cap v=\tilde{f}(v) \cap \Theta=\tilde{f}^{-1}(v) \cap v=\emptyset,
$$

então $\tilde{f}(v) \subset O_{D}$. Ainda, $\tilde{f}(\Theta) \cap O_{E}=\emptyset$. Desse modo, temos duas possibilidade para o conjunto $\Theta$ : 
1. $\tilde{f}(\Theta) \neq \Theta$;

2. $\tilde{f}(\Theta)=\Theta$.

O primeiro caso, implica que $\tilde{f}(\Theta) \cap \Theta=\emptyset$, pois $\Theta$ é uma componente conexa de um conjunto invariante. Logo,

$$
\tilde{f}(\Theta \cup v) \cap(\Theta \cup v)=\emptyset .
$$

Como $\tilde{f}(v) \subset O_{D}$ e $\tilde{f}(\Theta \cup v)$ é conexo temos $\tilde{f}(\Theta \cup v) \subset O_{D}$, portanto $\tilde{f}\left(O_{D}\right) \subset O_{D}$.

Suponha agora que $\tilde{f}(\Theta)=\Theta$. Desse modo, temos que $\tilde{f}\left(O_{D}\right)$ é uma componente conexa de $(\Theta \cup \tilde{f}(v))^{c}$. Se $\tilde{f}\left(O_{D}\right)$ não está contida em $O_{D}$, então $\tilde{f}\left(O_{D}\right) \cap O_{E} \neq \emptyset$. Logo $\tilde{f}(v) \cap O_{E} \neq \emptyset$, donde segue um absurdo. Assim, $\tilde{f}\left(O_{D}\right) \subset O_{D}$.

Para concluir o lema, resta mostrar que $\tilde{f}^{n}(v) \cap v=\emptyset$, para todo $n>0$. Sabemos que $\tilde{f}(v) \cap v=\emptyset$, logo, para todo $n \geq 2, \tilde{f}^{n}(v) \subset \tilde{f}\left(O_{D}\right)$ e esse não intercepta $v$. Nessas condições, $\tilde{f}^{n}(v) \cap v=\emptyset$, para todo $n>0$.

Observação 4.1.3. Pela escolha das constantes em 4.3 temos, para todo $\tilde{x} \in \mathbb{R}$, $\mu_{\text {trans }}(\tilde{x} \bmod 1)<M_{f}+M_{\text {Dehn }}+2$. Logo, para todo inteiro $n>0$,

$$
\tilde{f}^{n}\left(\{\tilde{x}\} \times\left[M_{f}+M_{D e h n}+2,+\infty[) \cap\{\tilde{x}\} \times\left[M_{f}+M_{D e h n}+2,+\infty[=\emptyset .\right.\right.\right.
$$

Como foi dito anteriormente, podemos fazer construções análogas para $\omega\left(B_{N}^{+}\right)$e obter que, para cada $\tilde{x} \in \mathbb{R}$,

$$
\left.\left.\left.\left.\tilde{f}^{n}(\{\tilde{x}\} \times]-\infty,-M_{f}-M_{\text {Dehn }}-2\right]\right) \cap\{\tilde{x}\} \times\right]-\infty,-M_{f}-M_{\text {Dehn }}-2\right]=\emptyset
$$

para todo inteiro $n>0$.

Seja $M>10\left(M_{f}+M_{\text {Dehn }}+2\right)$ e por 4.1. considere um ponto $\hat{z}_{M}$ tal que

$$
p_{2}\left(\hat{z}_{M}\right)<-M-1 \text { e } p_{2} \circ \hat{f}^{n_{0}}\left(\hat{z}_{M}\right)>M+1,
$$

para algum $n_{0}>0$. Considere $\tilde{g}$ um homeomorfismo do plano definido por

$$
\tilde{g}=\tilde{f}^{n_{0}}-(0,1) .
$$

Se $\tilde{g}$ tiver um ponto fixo, então $\tilde{g}\left(\tilde{z}_{\tilde{g}}\right)=\tilde{z}_{\tilde{g}}$, para algum $\tilde{z}_{\tilde{g}} \in \mathbb{R}^{2}$. Logo, $\tilde{f}^{n_{0}}\left(\tilde{z}_{\tilde{g}}\right)=$ $\tilde{z}_{\tilde{g}}+(0,1)$ e, portanto, $\rho_{v}\left(\tilde{z}_{\tilde{g}}\right)=1 / n_{0}$. O que é um absurdo, pois $\rho_{v}(\hat{f})=\{0\}$.

Assim, g̃ é uma aplicação de Brouwer, isto é, um homeomorfismo do plano sem pontos fixos. 
A ideia é construir uma decomposição do plano em tijolos livres e mostrar que essa possui uma cadeia fechada (ou periódica) de tijolos livres para $\tilde{g}$, donde seguirá uma contradição.

Como g̃ é o levantamento de uma aplicação do cilindro vertical podemos construir uma g̃-decomposição, invariante pela ação da translação horizontal

$$
(\tilde{x}, \tilde{y}) \longrightarrow(\tilde{x}+1, \tilde{y}) \text {. }
$$

Nessas condições, consideremos a faixa $[0,1] \times \mathbb{R}$ e iniciemos a decomposição com as seguintes afirmações:

Afirmação 4.1.4. Existe um inteiro positivo $n$ tal que o reticulado de $[0,1] \times[-M, M]$ com tijolos (ou blocos) de arestas de comprimento $1 / n$ é livre para $\tilde{g}$.

Demonstração. Suponha, por contradição, que para todo $n>1$ exista um tijolo não livre, denotado por $B_{n}$. Seja $\tilde{z}_{n}$ o centro de $B_{n}$. Como $\left(\tilde{z}_{n}\right)_{n \in \mathbb{N}^{*}} \subset[0,1] \times[-M, M]$, temos uma subsequência convergente para algum $\tilde{z} \in[0,1] \times[-M, M]$, digamos $\tilde{z}_{n_{k}} \longrightarrow \tilde{z}$ quando $k \longrightarrow+\infty$.

Sendo $\tilde{g}=\tilde{f}^{n}-(0,1)$ contínua e sem pontos fixos, temos que existe um $m_{0}>0$ tal que $\|\tilde{g}(\tilde{w})-\tilde{w}\| \geq m_{0}$ para todo $\tilde{w} \in[0,1] \times[-M, M]$. Portanto, sempre é possível construir um tijolo livre que contém $\tilde{z}$, isto é, existe $\delta>0$ tal que $B_{\delta}(\tilde{z})$ é livre por $\tilde{g}$. Mas, z̃ é o ponto de acumulação de $\left\{\tilde{z}_{n_{k}}\right\}_{n_{k} \in \mathbb{N}_{1}}$ e $\operatorname{diam}\left(B_{n_{k}}\right) \longrightarrow 0$, quando $k \longrightarrow+\infty$. Assim, existe $N>1$ tal que para todo $n_{k}>N$,

$$
B_{n_{k}} \subset B_{\delta}(\tilde{z}) \text {. }
$$

Donde vem um absurdo, pois $\tilde{g}\left(B_{n_{k}}\right) \cap B_{n_{k}} \neq \emptyset$ para todo $n_{k}>1$.

Afirmação 4.1.5. Existe um inteiro positivo $n$ tal que as faixas verticais ilimitadas de $[0,1] \times[M,+\infty[$ e de $[0,1] \times]-\infty,-M-1]$ com bases de comprimento $1 / n$ sejam tijolos livres para $\tilde{g}$.

Demonstração. Suponha, por contradição, que para todo $n>1$ exista um tijolo não livre, denotado por $B_{n}^{+}$.

Seja $\tilde{z}_{n} \in([0,1] \times\{M\}) \cap B_{n}^{+}$. Como $\left(\tilde{z}_{n}\right)_{n \in \mathbb{N}^{*}} \subset[0,1] \times\{M\}$, temos uma subsequência convergente para algum $\tilde{z} \in[0,1] \times\{M\}$.

Por construção de $v, \tilde{f}^{n}(v) \bigcap v=\emptyset$ para todo inteiro $n>0$, e pela escolha da constante $M$ (e observação 4.1.1),

$$
v_{\tilde{z}}=\left\{p_{1}(\tilde{z})\right\} \times[M,+\infty[
$$


é tal que,

$$
\tilde{f}^{n_{0}}\left(v_{\tilde{z}}\right) \bigcap v_{\tilde{z}}=\emptyset
$$

Assim, $\left(\tilde{f}^{n_{0}}\left(v_{\tilde{z}}\right)-(0,1)\right) \bigcap\left(v_{\tilde{z}}-(0,1)\right)=\emptyset$, ou seja, $\tilde{g}\left(v_{\tilde{z}}\right) \bigcap\left(v_{\tilde{z}}-(0,1)\right)=\emptyset$. Portanto,

$$
\tilde{g}\left(v_{\tilde{z}}\right) \bigcap v_{\tilde{z}}=\emptyset \text {. }
$$

Nessas condições, existe $\epsilon>0$ tal que $\left.V_{\epsilon}\left(v_{\tilde{z}}\right)=\right] p_{1}(\tilde{z})-\epsilon, p_{1}(\tilde{z})+\epsilon[\times[M,+\infty$ [ é livre por $\tilde{g}$, isto é, $\tilde{g}\left(V_{\epsilon}\left(v_{\tilde{z}}\right)\right) \cap V_{\epsilon}\left(v_{\tilde{z}}\right)=\emptyset$. Mas da existência de $n_{i}$ tal que $B_{n_{i}}^{+} \subset V_{\epsilon}\left(v_{\tilde{z}}\right)$ e $\tilde{g}\left(B_{n_{i}}^{+}\right) \cap B_{n_{i}}^{+} \neq \emptyset$, temos uma contradição que demonstra a afirmação.

A obtenção dos outros tijolos livres do plano é feita pela translação horizontal. Nessas condições, temos que existe um inteiro $N>0$ tal que para todo inteiro $n$, os conjuntos

$$
\left.\left.F_{n}^{-}=[n / N,(n+1) / N] \times\right]-\infty,-M-1\right]
$$

e

$$
F_{n}^{+}=[n / N,(n+1) / N] \times[M+1,+\infty[
$$

são livres por $\tilde{g}$, isto é,

$$
\tilde{g}\left(F_{n}^{+}\right) \cap F_{n}^{+}=\emptyset \text { e } \tilde{g}\left(F_{n}^{-}\right) \cap F_{n}^{-}=\emptyset \text {, para todo inteiro } n .
$$

Supondo como antes que a constante de Dehn twist, $m$, seja positiva, temos que existe um $K_{c r i t}>0$, tal que para todo inteiro $n$,

$$
\tilde{g}\left(F_{n}^{+}\right) \cap F_{l}^{+} \neq \emptyset, \text { para todo } l \geq n+K_{\text {crit }}
$$

e

$$
\tilde{g}\left(F_{n}^{-}\right) \cap F_{l}^{-} \neq \emptyset, \text { para todo } l \leq n-K_{\text {crit }} .
$$

Para finalizar o teorema basta mostrar que essa $\tilde{g}$-decomposição possui uma cadeia fechada de tijolos livres, donde seguirá uma contradição, do lema 3.4.1 com o fato de $\tilde{g}$ não ter pontos fixos. Veja a figura 4.2 abaixo (os pontilhados são a imagem por $\tilde{g}$ dos tijolos, indicados pelas setas).

Suponha que $\tilde{z}_{M} \in F_{0}^{-}$onde $\tilde{z}_{M}$ é o ponto tal que $p_{2} \circ \tilde{g}\left(\tilde{z}_{M}\right)>M+1$. Assim, existe $F_{l_{0}}^{+}$um tijolo tal que $F_{l_{0}}^{+} \cap \tilde{g}\left(F_{0}^{-}\right) \neq \emptyset$, para algum inteiro $l_{0}$. Como $\rho_{v}(\hat{f})=\{0\}$, segue da definição de $\tilde{g}$ que existe um ponto $\hat{w}_{M} \in S^{1} \times[M,+\infty[$ tal que

$$
p_{2}\left(\hat{g}^{n}(\hat{w})\right) \longrightarrow-\infty \text {, quando } n \longrightarrow+\infty
$$


onde $\hat{g}$ é a projeção de $\tilde{g}$ para $S^{1} \times \mathbb{R}$.

Nessas condições, podemos escolher um ponto $\tilde{w} \in \pi^{-1}(\hat{w})$ tal que $\tilde{w} \in F_{l_{1}}^{+}$satisfazendo:

- $l_{1}>l_{0}+K_{c r i t}$, então $\tilde{g}\left(F_{l_{0}}^{+}\right) \cap F_{l_{1}}^{+} \neq \emptyset$;

- $\tilde{g}^{n_{1}}(\tilde{w}) \in F_{l_{2}}^{-}$para algum inteiro $n_{1}>0$ e $l_{2}>K_{\text {crit }}$.

Como $\tilde{g}\left(F_{l_{2}}^{-}\right) \cap F_{0}^{-} \neq \emptyset$, temos uma cadeia fechada de tijolos livre, digamos

$$
F_{0}^{-}, F_{l_{0}}^{+}, F_{l_{1}}^{+}, F_{1}, F_{2}, \ldots, F_{n_{1}-1}, F_{l_{2}}^{-}, F_{0}^{-},
$$

onde $F_{i}$ é um tijolo livre de $\mathbb{R} \times[-M-1, M+1]$, para $i=1,2, \ldots, n_{1}-1$. Portanto, como tínhamos dito antes, isso é um absurdo, pois a existência de um ponto fixo para $\tilde{g}$ implica que existe um ponto com número de rotação vertical positivo para $\hat{f}$.

Desse modo, existe $M>0$ tal que

$$
\hat{f}^{n}\left(S^{1} \times[0,1]\right) \subset S^{1} \times[-M, M]
$$

para todo inteiro $n>0$, e, nessas condições, temos que existe um continuum essencial invariante por $\hat{f}$. 


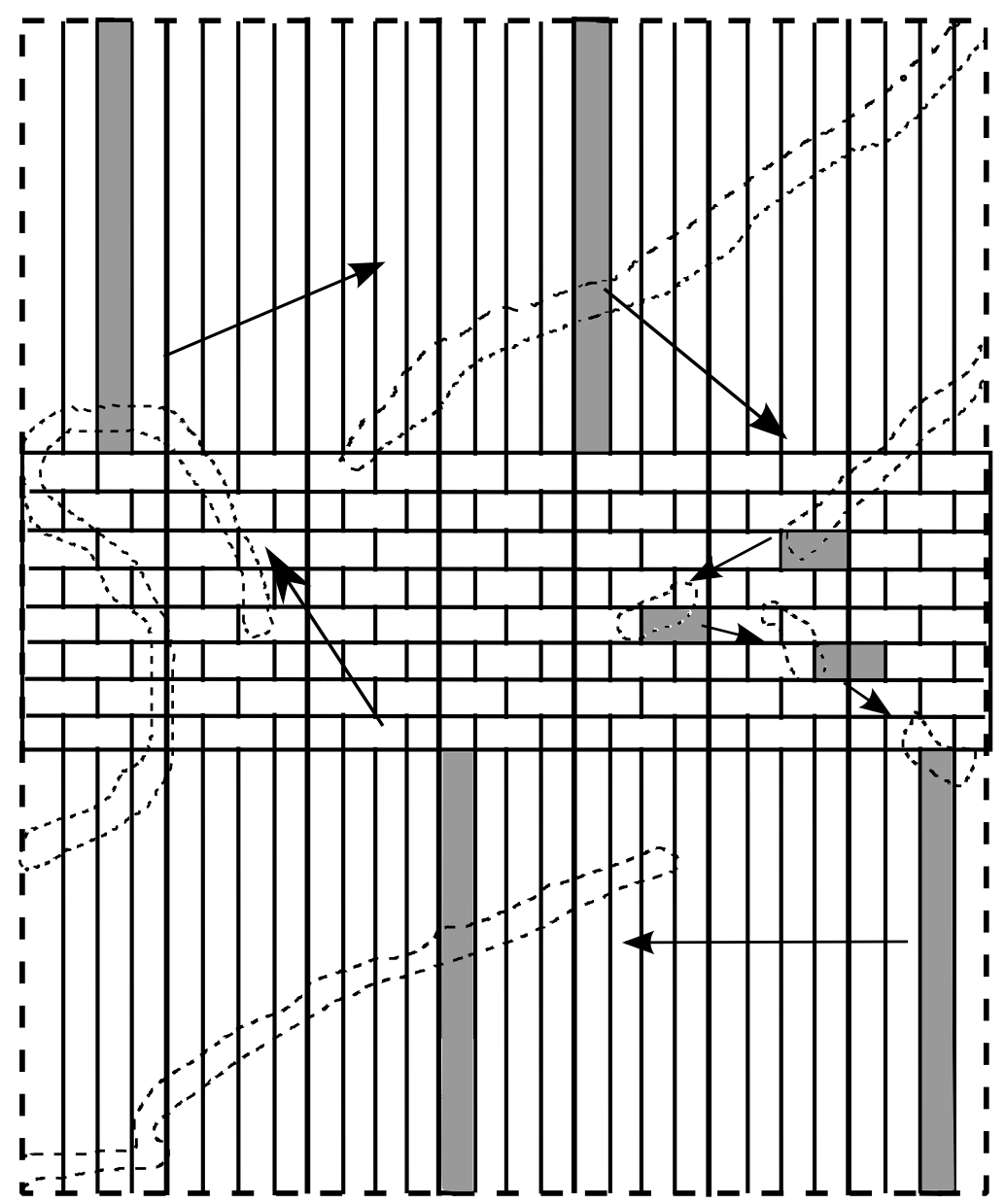

Figura 4.2: Decomposição do Plano por Tijolos Livres para $\tilde{g}$.

Os corolários seguintes são quase imediatos do teorema anterior.

Corolário 4.1.6. Sejam $f \in D T\left(\mathbb{T}^{2}\right)$ e um levantamento $\hat{f} \in D T\left(S^{1} \times \mathbb{R}\right)$ tal que $\rho_{v}(\hat{f})=[a, p / q]$, onde $a$ é qualquer número real menor ou igual que o racional $p / q$. Então, existe $M>0$ tal que para todo $\hat{z} \in S^{1} \times \mathbb{R}, p_{2} \circ \hat{f}^{n}(\hat{z})-p_{2}(\hat{z})-n p / q<M$, para todo inteiro $n>0$.

Demonstração. Sem perda de generalidade, se considerarmos $\hat{f}^{q}-(0, p)$, podemos supor que $\rho_{v}(\hat{f})=[a, 0]$, para algum $a \leq 0$.

O teorema 3.1.2 implica que $B_{N}^{+} \neq \emptyset, B_{S}^{-} \neq \emptyset, B_{S}^{-}($inv $) \neq \emptyset$ e $B_{N}^{+}($inv $) \neq \emptyset$.

Suponha, por um instante, que $\omega\left(B_{S}^{-}\right)=\emptyset$. Assim, pelo lema 3.2.3. $\omega\left(B_{S}^{-}(\right.$inv $\left.)\right)=\emptyset$ e, desse modo, pelo lema 3.2 .2 , existe $\epsilon>0$ tal que $\rho_{v}\left(\hat{f}^{-1}\right) \supset[-\epsilon, 0]$, ou seja, $\rho_{v}(\hat{f}) \supset$ 
$[0, \epsilon]$, um absurdo.

Nessas condições, temos $\omega\left(B_{S}^{-}\right) \neq \emptyset \mathrm{e} \omega\left(B_{N}^{+}\right) \neq \emptyset$. Portanto, se assumirmos que para todo $M>0$ existem um inteiro $n>0$ e um ponto $\hat{z} \in S^{1} \times \mathbb{R}$ tal que

$$
p_{2} \circ \hat{f}^{n}(\hat{z})-p_{2}(\hat{z})>M,
$$

e usarmos exatamente as ideias apresentadas no teorema anterior, obteremos que existe um ponto com número de rotação vertical positivo, uma contradição que prova o corolário.

Corolário 4.1.7. Sejam $f \in D T\left(\mathbb{T}^{2}\right)$ e um levantamento $\hat{f} \in D T\left(S^{1} \times \mathbb{R}\right)$. Então existe $M>0$ tal que se para $\hat{z}_{1}, \hat{z}_{2} \in S^{1} \times \mathbb{R}$ ocorrer

$$
p_{2} \circ \hat{f}^{n_{1}}\left(\hat{z}_{1}\right)-p_{2}\left(\hat{z}_{1}\right)>M
$$

$\mathrm{e}$

$$
p_{2} \circ \hat{f}^{n_{2}}\left(\hat{z}_{2}\right)-p_{2}\left(\hat{z}_{2}\right)<-M,
$$

$\operatorname{com} n_{1}, n_{2} \in \mathbb{N}^{*}$, então $0 \in \operatorname{int}\left(\rho_{v}(\hat{f})\right)$.

Demonstração. Como no teorema anterior, fixemos $\tilde{f} \in D T\left(\mathbb{R}^{2}\right)$ e consideremos $M>$ $10\left(M_{f}+M_{\text {Dehn }}+2\right)$. Suponha que $\hat{f}$ não tenha pontos fixos. Assim, segue do teorema 2.2.6 que existe uma curva fechada simples homotopicamente não trivial $\gamma \subset S^{1} \times \mathbb{R}$ tal que $\hat{f}(\gamma) \cap \gamma=\emptyset \mathrm{e} \gamma \subset S^{1} \times\left[-m_{D}, m_{D}\right]$, onde $m_{D}>0$ é o menor número real tal que satisfaça

$$
\tilde{f}\left(\{\tilde{x}\} \times\left[m_{D},+\infty[) \subset[\tilde{x}+10,+\infty[\times \mathbb{R}\right.\right.
$$

e

$$
\left.\left.\left.\left.\tilde{f}(\{\tilde{x}] \times]-\infty,-m_{D}\right]\right) \subset\right]-\infty, \tilde{x}-10\right] \times \mathbb{R},
$$

para todo $\tilde{x} \in \mathbb{R}$. Um simples cálculo mostra que $M>2 m_{D}+10$ e isso contradiz as hipóteses do corolário. Portanto, $\hat{f}$ tem um ponto fixo e dai $0 \in \rho_{v}(\hat{f})$.

O teorema 3.1.2 implica que $B_{N}^{+} \neq \emptyset$ e $B_{S}^{-} \neq \emptyset$ e o mesmo ocorre para a inversa de $\hat{f}$, isto é, $B_{N}^{+}($inv $) \neq \emptyset$ e $B_{S}^{-}($inv $) \neq \emptyset$. Se $\omega\left(B_{N}^{+}\right)=\emptyset$, então, pelo lema 3.2.2. existe $\delta>0$ tal que $\rho_{v}(\hat{f}) \supset[0, \delta]$. Do lema 3.2.3, obtemos que se $\omega\left(B_{N}^{+}(i n v)\right)=\emptyset$ então, existe $\epsilon>0$ tal que $\rho_{v}\left(\hat{f}^{-1}\right) \supset[0, \epsilon]$, donde temos $\rho_{v}(\hat{f}) \supset[-\epsilon, \delta]$ e isso conclui a tese do corolário. Nessas condições, podemos supor que $\omega\left(B_{N}^{+}\right) \neq \emptyset$ e $\omega\left(B_{S}^{-}\right) \neq \emptyset$. Procedendo como na prova do corolário anterior obtemos uma contradição se $\rho_{v}(\hat{f})=[a, 0]$, para algum $a \leq 0$, ou $\rho_{v}(\hat{f})=[0, b]$, para algum $b \geq 0$. Isso finaliza o corolário. 


\subsection{Demonstração do teorema 1.3.1}

Considere $f \in D T\left(\mathbb{T}^{2}\right)$ que preserva área e que tenha um levantamento $\hat{f} \in D T\left(S^{1} \times \mathbb{R}\right)$ com fluxo zero. Decorre do teorema 2.2.6 que $\hat{f}$ tem ponto fixo. Quer-se mostrar a tese,

$$
\rho_{v}(\hat{f})=\left\{\begin{array}{l}
\{0\} \\
{[\mathrm{a}, \mathrm{b}] \quad \operatorname{com} a<0<b .}
\end{array}\right.
$$

Temos duas possibilidades:

1. $\bigcup_{n \geq 0} \hat{f}^{n}(H)$ é limitado;

2. $\bigcup_{n \geq 0} \hat{f}^{n}(H)$ é ilimitado.

No primeiro caso, temos que $\bigcup_{n \in \mathbb{Z}} \hat{f}^{n}\left(H^{-}\right)$é limitado superiormente e invariante por $\hat{f}, \operatorname{logo}$ existe uma componente conexa de seu bordo que é compacta, invariante e que separa os fins do cilindro. Assim, $\rho_{v}(\hat{f})=\{0\}$.

No segundo caso, temos que

$$
\bigcup_{n \geq 0} \hat{f}^{n}(H)
$$

é ilimitado em ambas as direções. De fato, suponha que $\bigcup_{n \geq 0} \hat{f}^{n}(H)$ esteja contido em $S^{1} \times\left[-M_{0},+\infty\left[\right.\right.$, com $M_{0}>0$.

Considere o conjunto aberto

$$
O:=\bigcup_{n \geq 0} \hat{f}^{n}\left(H^{+}\right)
$$

Temos que $O$ é positivamente invariante e que existe um conjunto $K \subset \partial O$ compacto, conexo e que separa o cilindro em duas componentes. Desse modo, se $K^{+}$é a componente conexa de $K^{c}$ que contém o fim superior,

$$
\hat{f}(K) \subset \operatorname{fecho}\left(K^{+}\right) \text {. }
$$

Se $\hat{f}(K)=K$, então estamos no caso 1 , o que é um absurdo. Assim,

$$
\hat{f}\left(f e c h o\left(K^{+}\right)\right) \subsetneq f e c h o\left(K^{+}\right) .
$$

O que contradiz o fato de $\hat{f}$ ter fluxo zero. Veja a figura abaixo. 


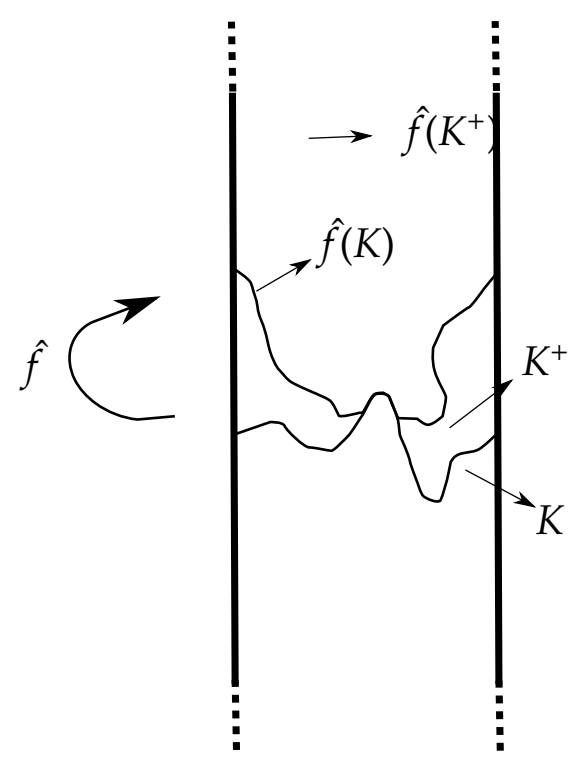

Figura 4.3: Violação do fluxo zero.

Dessa feita, $\bigcup_{n \geq 0} \hat{f}^{n}(H)$ é ilimitado superiormente e inferiormente. Portanto, dado $M>1$ existem $\hat{z}_{1}, \hat{z}_{2} \in S^{1} \times \mathbb{R}$ e $n_{1}, n_{2} \in \mathbb{N}^{*}$ tais que

$$
p_{2} \circ \hat{f}^{n_{1}}\left(\hat{z}_{1}\right)-p_{2}\left(\hat{z}_{1}\right)>M
$$

e

$$
p_{2} \circ \hat{f}^{n_{2}}\left(\hat{z}_{2}\right)-p_{2}\left(\hat{z}_{2}\right)<-M \text {. }
$$

Pelo corolário 1.3 .4 (anterior), $0 \in \operatorname{int}\left(\rho_{v}(\hat{f})\right)$. 
[1] Addas-Zanata S. (2000): On the dynamics of twist maps of the torus. Doctoral Thesis IME-USP. (In Portuguese).

[2] Addas-Zanata S. (2002): On the existence of a new type of periodic and quasiperiodic orbits for twist maps of torus. Nonlinearity 15, 1399-1416 7.

[3] Addas-Zanata S. (2005): Some extensions of the Poincaré-Birkhoff theorem to the cylinder and remark on mappings of the torus homotopic to Dehn twist. Nonlinearity 18, 2243-2260.

[4] Addas-Zanata S. and Tal F. A. (2009): Homeomorphisms of the annulus with a transitive lift. Math. Zeit..

[5] Addas-Zanata S. and Tal F. A. (2010): On generic rotationless diffeomorphisms of the annulus. Proc. Am. Math. Soc. 138 1023-1031.

[6] Addas-Zanata S., Garcia B. and Tal F. A. (2010): On the dynamics of homeomorphisms of the torus homotopic to Dehn twists. preprint.

[7] Doeff E (1997): Rotation measures for homeomorphisms of the torus homotopic to a Dehn twist. Ergod. Theor. Dynam. Syst. 17, 1-17.

[8] Doeff E and Misiurewicz M (1997): Shear rotation numbers. Nonlinearity 10, 1755-1762.

[9] Franks J (1988): Recurrence and fixed points of surface homeomorphisms. Ergod. Theor. Dynam. Syst. 8 99-107. 
[10] Franks J (1988): Generalizations of the Poincaré-Birkhoff theorem. Ann. of Math. 128 139-151.

[11] Fathi A, Laudenbach F and Poenaru V (1979): Travaux de Thurston sur les surfaces. Astérisque 66-67 1-284.

[12] Llibre J. and Mackay R. (1991): Rotation vectors and entropy for homeomorphisms of the torus isotopic to the identity. Erg. Th. Dyn. Sys. 11 115-128.

[13] Le Calvez P (1991): Propriétés dynamiques des difféomorphismes de l' anneau et du tore. Astérisque 204 1-131.

[14] Le Calvez P (1990): Etude topologique des applications déviant la vertical. Ensaios Matemáticos, Soc. Brasileira de Matemática Vol. 2.

[15] Le Roux F. (2004): Homéomorphismes de surfaces: théorèmes de la fleur de Leau-Fatou et de la variété stable. Astérisque 292210 pp.

[16] Béguin F., Le Roux F. (2007): Dynamique topologique sur les surface.

[17] M. Bonino.: Around Brouwer theory of fixed point free planar homeomorphisms. Notes de cours, Grenoble, juin 2006.

[18] Misiurewicz M and Ziemian K (1989): Rotation sets for maps of tori. J. London Math. Soc. 40 490-506.

[19] Brown M (1984): A new proof of Brouwer's lemma on translation arcs. Houston J. Math 10 35-41.

[20] Kerekjarto B (1928-29): The plane translation theorem of Brouwer and the last geometric theorem of Poincaré. Acta Sci. Math (Szaged.) 4 86-102.

[21] Epstein D (1966): Curves on 2-manifolds and isotopies. Acta Math. Soc. 115 83-107.

[22] Birkhoff G. D. (1935): Nouvelles Recherches sur les systèmes dynamiques. Collected Math. Papers Vol. 2 530-661.

[23] Angenent, S. B., (1990) Monotone recurrence relations, their Birkhoff orbits and topological entropy. Ergod. Theor. Dynam. Syst. 10 15-41. 
[24] Katok, A. and Hasselblatt, B. Introduction to the modern theory of dynamical systems, Encyclopedia of Mathematics and its Apllications 54, Cambridge University Press, Cambridge (1995).

[25] Carneiro, M. J. D., Ragazzo, C. G., Zanata, S. A., Introdução à Dinâmica de Aplicações do Tipo Twist, 25 Colóquio Brasileiro de Matemática, 2005.

[26] De Melo, W., van Strien, S. One-Dimensional Dynamics, Springer-Verlag, New York (1993).

[27] Devaney, R.L. An Introduction to chaotic dynamical systems, Second Edition, Addison Wesley, Redwood, CA (1989).

[28] Hubbard, J. H. Teichmüller Theory and Applications to Geometry, Topology, and Dynamics. Vol. 1, Matrix Editions, Ithaca, New York, 2006.

[29] Alsed'a, Li., Llibre, J. and Misiurewicz, M. Combinatorial dynamics and entropy in dimension one, Second Edition, World Scientific (Advanced Series in Nonlinear Dynamics, vol. 5), Singapore, 2000.

[30] Walters, P. An Introduction to Ergodic Theory. New York, Springer-Verlag, (2000).

[31] Moser J. e Siegel K. Lectures on celestial mechanics, Springer-Verlag, NY (1971). 
